This is an Open Access article, distributed under the terms of the Creative Commons Attribution licence (http://creativecommons.org/licenses/by/4.0/), which permits unrestricted re-use, distribution, and reproduction in any medium, provided the original work is properly cited.

doi:10.1017/etds.2019.105

\title{
A construction of subshifts and a class of semigroups
}

\author{
TOSHIHIRO HAMACHI $\dagger$ and WOLFGANG KRIEGER $\ddagger$ \\ $\dagger$ Faculty of Mathematics, Kyushu University, 744 Motooka, Nishi-ku, \\ Fukuoka 819-0395, Japan \\ (e-mail: t.hamachi.796@m.kyushu-u.ac.jp) \\ $\ddagger$ Institute for Applied Mathematics, University of Heidelberg, Im Neuenheimer Feld 205, \\ 69120 Heidelberg, Germany \\ (e-mail: krieger@math.uni-heidelberg.de)
}

(Received 19 November 2018 and accepted in revised form 23 October 2019)

Abstract. Subshifts with property $(A)$ are constructed from a class of directed graphs. As special cases the Markov-Dyck shifts are shown to have property $(A)$. The semigroups that are associated to $\mathcal{R}$-graph shifts with Property $(A)$ are determined.

Key words: symbolic dynamics, $\mathcal{R}$-graph shift, Property $(A)$, semigroup 2010 Mathematics Subject Classification: 37B10 (Primary); 20M05 (Secondary)

\section{Introduction}

Let $\Sigma$ be a finite alphabet and let $S$ be the shift on the shift space $\Sigma^{\mathbb{Z}}$,

$$
S\left(\left(x_{i}\right)_{i \in \mathbb{Z}}\right)=\left(x_{i+1}\right)_{i \in \mathbb{Z}}, \quad\left(x_{i}\right)_{i \in \mathbb{Z}} \in \Sigma^{\mathbb{Z}} .
$$

An $S$-invariant closed subset $X$ of $\Sigma^{\mathbb{Z}}$ is called a subshift. For an introduction to the theory of subshifts, see $[\mathbf{K i}]$ or $[\mathbf{L M}]$. In [Kr2], a Property $(A)$ of subshifts was introduced that is an invariant of topological conjugacy. Also, in [Kr2], there was constructed for a subshift $X$ with Property $(A)$ a semigroup $\mathcal{S}(X)$ that is invariantly attached to $X$. Prototypes of subshifts with Property $(A)$ are the Dyck shifts [Kr1]. To recall the construction of the Dyck shifts, let $N>1$ and let $\alpha^{-}(n), \alpha^{+}(n), 0 \leq n<N$, be the generators of the Dyck inverse monoid (the polycyclic monoid $[\mathbf{N P}]$ ) $\mathcal{D}_{N}$ that satisfy the relations

$$
\alpha^{-}(n) \alpha^{+}(m)= \begin{cases}1 & \text { if } n=m, \\ 0 & \text { if } n \neq m\end{cases}
$$


The Dyck shifts are defined as the subshifts

$$
D_{N} \subset\left(\left\{\alpha^{-}(n): 0 \leq n<N\right\} \cup\left\{\alpha^{+}(n): 0 \leq n<N\right\}\right)^{\mathbb{Z}}
$$

with the admissible words $\left(\sigma_{i}\right)_{1 \leq i \leq I}, I \in \mathbb{N}$, of $D_{N}, N>1$, given by the condition

$$
\prod_{1 \leq i \leq I} \sigma_{i} \neq 0
$$

The semigroup $\mathcal{S}\left(D_{N}\right)$ is the Dyck inverse monoid $\mathcal{D}_{N}, N>1$.

We denote a finite directed graph with vertex set $\mathfrak{P}$ and edge set $\mathcal{E}$ by $G(\mathfrak{P}, \mathcal{E})$. As notation for the source (target) vertex of an edge or directed path in a directed graph, we use $s(t)$. We recall from [Kr4] the notion of an $\mathcal{R}$-graph. Let there be given a finite directed graph $G(\mathcal{V}, \mathcal{E})$. Assume also that we are given a partition

$$
\mathcal{E}=\mathcal{E}^{-} \cup \mathcal{E}^{+} .
$$

We set

$$
\begin{aligned}
& \mathcal{E}^{-}(\mathfrak{q}, \mathfrak{r})=\left\{e^{-} \in \mathcal{E}^{-}: s\left(e^{-}\right)=\mathfrak{q}, t\left(e^{-}\right)=\mathfrak{r}\right\}, \\
& \mathcal{E}^{+}(\mathfrak{q}, \mathfrak{r})=\left\{e^{+} \in \mathcal{E}^{+}: s\left(e^{+}\right)=\mathfrak{r}, t\left(e^{+}\right)=\mathfrak{q}\right\}, \quad \mathfrak{q}, \mathfrak{r} \in \mathfrak{P} .
\end{aligned}
$$

We assume that $\mathcal{E}^{-}(\mathfrak{q}, \mathfrak{r}) \neq \emptyset$ if and only if $\mathcal{E}^{+}(\mathfrak{q}, \mathfrak{r}) \neq \emptyset, \mathfrak{q}, \mathfrak{r} \in \mathfrak{P}$, and we assume that the directed graph $G\left(\mathfrak{P}, \mathcal{E}^{-}\right)$is strongly connected or, equivalently, that the directed graph $G\left(\mathfrak{P}, \mathcal{E}^{+}\right)$is strongly connected. We call $G\left(\mathfrak{P}, \mathcal{E}^{-} \cup \mathcal{E}^{+}\right)$a partitioned directed graph. Let there further be given relations $\dagger$

$$
\mathcal{R}(\mathfrak{q}, \mathfrak{r}) \subset \mathcal{E}^{-}(\mathfrak{q}, \mathfrak{r}) \times \mathcal{E}^{+}(\mathfrak{q}, \mathfrak{r}), \quad \mathfrak{q}, \mathfrak{r} \in \mathfrak{P},
$$

and set

$$
\mathcal{R}=\bigcup_{\mathfrak{q}, \mathfrak{r} \in \mathfrak{P}} \mathcal{R}(\mathfrak{q}, \mathfrak{r}) .
$$

The resulting structure, that we call an $\mathcal{R}$-graph, we denote by $G_{\mathcal{R}}\left(\mathfrak{P}, \mathcal{E}^{-} \cup \mathcal{E}^{+}\right)$. We also recall the construction of a semigroup (with zero) $\mathcal{S}\left(G_{\mathcal{R}}\left(\mathfrak{P}, \mathcal{E}^{-} \cup \mathcal{E}^{+}\right)\right.$) from an $\mathcal{R}$-graph $G_{\mathcal{R}}\left(\mathfrak{P}, \mathcal{E}^{-} \cup \mathcal{E}^{+}\right)$as described in $[\mathbf{K r} 3]$. The semigroup $\mathcal{S}\left(G_{\mathcal{R}}\left(\mathfrak{P}, \mathcal{E}^{-} \cup \mathcal{E}^{+}\right)\right)$contains idempotents $\mathbf{1}_{\mathfrak{p}}, \mathfrak{p} \in \mathfrak{P}$, and has $\mathcal{E}$ as a generating set. Besides $\mathbf{1}_{\mathfrak{p}}^{2}=\mathbf{1}_{\mathfrak{p}}, \mathfrak{p} \in \mathfrak{P}$, the defining relations are

$$
f^{-} g^{+}= \begin{cases}\mathbf{1}_{\mathfrak{q}} & \text { if } f^{-} \in \mathcal{E}^{-}(\mathfrak{q}, \mathfrak{r}), g^{+} \in \mathcal{E}^{+}(\mathfrak{q}, \mathfrak{r}),\left(f^{-}, g^{+}\right) \in \mathcal{R}(\mathfrak{q}, \mathfrak{r}), \mathfrak{q}, \mathfrak{r} \in \mathfrak{P} \\ 0 & \text { if } f^{-} \in \mathcal{E}^{-}(\mathfrak{q}, \mathfrak{r}), g^{+} \in \mathcal{E}^{+}(\mathfrak{q}, \mathfrak{r}),\left(f^{-}, g^{+}\right) \notin \mathcal{R}(\mathfrak{q}, \mathfrak{r}), \mathfrak{q}, \mathfrak{r} \in \mathfrak{P} \\ 0 & \text { if } f^{-} \in \mathcal{E}^{-}(\mathfrak{q}, \mathfrak{r}), g^{+} \in \mathcal{E}^{+}\left(\mathfrak{q}^{\prime}, \mathfrak{r}\right), \mathfrak{q}, \mathfrak{q}^{\prime}, \mathfrak{r} \in \mathfrak{P}, \mathfrak{q} \neq \mathfrak{q}^{\prime}\end{cases}
$$

and

$$
\begin{array}{ll}
\mathbf{1}_{\mathfrak{q}} e^{-}=e^{-} \mathbf{1}_{\mathfrak{r}}=e^{-}, & e^{-} \in \mathcal{E}^{-}(\mathfrak{q}, \mathfrak{r}), \\
\mathbf{1}_{\mathfrak{r}} e^{+}=e^{+} \mathbf{1}_{\mathfrak{q}}=e^{+}, & e^{+} \in \mathcal{E}^{+}(\mathfrak{q}, \mathfrak{r}), \quad \mathfrak{q}, \mathfrak{r} \in \mathfrak{P},
\end{array}
$$

and

$$
\mathbf{1}_{\mathfrak{q}} \mathbf{1}_{\mathfrak{r}}=0, \quad \mathfrak{q}, \mathfrak{r} \in \mathfrak{P}, \quad \mathfrak{q} \neq \mathfrak{r} .
$$

$\dagger$ We consider complete heterogeneous relations. 
We call $\mathcal{S}\left(G_{\mathcal{R}}\left(\mathfrak{P}, \mathcal{E}^{-} \cup \mathcal{E}^{+}\right)\right)$an $\mathcal{R}$-graph semigroup. We write $\mathcal{S}^{-}\left(G_{\mathcal{R}}\left(\mathfrak{P}, \mathcal{E}^{-} \cup \mathcal{E}^{+}\right)\right)$ $\left(\mathcal{S}^{+}\left(G_{\mathcal{R}}\left(\mathfrak{P}, \mathcal{E}^{-} \cup \mathcal{E}^{+}\right)\right)\right)$for the set of non-zero elements of the subsemigroup of $\mathcal{S}\left(G_{\mathcal{R}}\left(\mathfrak{P}, \mathcal{E}^{-} \cup \mathcal{E}^{+}\right)\right)$that is generated by $\mathcal{E}^{-}\left(\mathcal{E}^{+}\right)$.

Special cases are the graph inverse semigroups of finite directed graphs $G(\mathfrak{P}, \mathcal{E})$ (see $[\mathbf{A H}]$ and $[\mathbf{L}, \S 18.7]$ and compare $[\mathbf{C K}])$. With the edge set $\mathcal{E}^{-}=\left\{e^{-}: e_{\circ} \in \mathcal{E}_{\circ}\right\}$ of a copy of $G(\mathfrak{P}, \mathcal{E})$, and with the edge set $\mathcal{E}^{+}=\left\{e^{+}: e \in \mathcal{E}\right\}$ of the reversal of $G(\mathfrak{P}, \mathcal{E})$, the graph inverse semigroup $\mathcal{S}(G(\mathfrak{P}, \mathcal{E}))$ of $G(\mathfrak{P}, \mathcal{E})$ is the $\mathcal{R}$-graph semigroup of the partitioned graph $G\left(\mathfrak{P}, \mathcal{E}^{-} \cup \mathcal{E}^{+}\right)$with the relations

$$
\mathcal{R}(\mathfrak{q}, \mathfrak{r})=\left\{\left(e^{-}, e^{+}\right): e \in \mathcal{E}, s(e)=\mathfrak{q}, t(e)=\mathfrak{r}\right\}, \quad \mathfrak{q}, \mathfrak{r} \in \mathfrak{P} .
$$

In $[\mathbf{H I}]$, a criterion was given for the existence of an embedding of an irreducible subshift of finite type into a Dyck shift and this result was extended in [HIK] to a larger class of target shifts with Property $(A)$. These target shifts were constructed by a method that presents the subshifts by means of a suitably structured irreducible finite labelled directed graph with labels taken from the inverse semigroup of an irreducible finite directed graph, in which every vertex has at least two incoming edges. This method was extended in [Kr4] by the use of $\mathcal{R}$-graph semigroups. Following [HIK, Kr4], we describe this construction.

We denote a finite labelled directed graph with vertex set $\mathcal{V}$, edge set $\Sigma$ and a label map $\lambda$ by $G(\mathcal{V}, \Sigma, \lambda)$. Let there be given an $\mathcal{R}$-graph $G_{\mathcal{R}}=G_{\mathcal{R}}\left(\mathfrak{P}, \mathcal{E}^{-} \cup \mathcal{E}^{+}\right)$and a finite strongly connected labelled directed graph $G(\mathcal{V}, \Sigma, \lambda)$ such that:

(G1) $\lambda(\sigma) \in S^{-}\left(G_{\mathcal{R}}\right) \cup\left\{\mathbf{1}_{\mathfrak{p}}: \mathfrak{p} \in \mathfrak{P}\right\} \cup S^{+}\left(G_{\mathcal{R}}\right), \sigma \in \Sigma$.

The label map $\lambda$ extends to finite paths $\left(\sigma_{i}\right)_{1 \leq i \leq I}$ in the graph $G(\mathcal{V}, \Sigma)$ by

$$
\lambda\left(\left(\sigma_{i}\right)_{1 \leq i \leq I}\right)=\prod_{1 \leq i \leq I} \lambda\left(\sigma_{i}\right) .
$$

Denoting for $\mathfrak{p} \in \mathfrak{P}$ by $\mathcal{V}(\mathfrak{p})$ the set of $V \in \mathcal{V}$ such that there is a cycle $\left(\sigma_{i}\right)_{1 \leq i \leq I}$, $I \in \mathbb{N}$, in the graph $G(\mathcal{V}, \Sigma)$ from $V$ to $V$ such that

$$
\lambda\left(\left(\sigma_{i}\right)_{1 \leq i \leq I}\right)=\mathbf{1}_{\mathfrak{p}}
$$

we require the following Conditions (G2)-(G5) to be satisfied.

(G2) $\mathcal{V}(\mathfrak{p}) \neq \emptyset, \mathfrak{p} \in \mathfrak{P}$.

$(\mathrm{G} 3)\{\mathcal{V}(\mathfrak{p}): \mathfrak{p} \in \mathfrak{P}\}$ is a partition of $\mathcal{V}$.

(G4) For $V \in \mathcal{V}(\mathfrak{p}), \mathfrak{p} \in \mathfrak{P}$, and for all edges $\sigma$ that leave $V, \mathbf{1}_{\mathfrak{p}} \lambda(\sigma) \neq 0$, and for all edges $\sigma$ that enter $V, \lambda(\sigma) \mathbf{1}_{\mathfrak{p}} \neq 0$.

(G5) For $f \in \mathcal{S}\left(G_{\mathcal{R}}\left(\mathfrak{P}, \mathcal{E}^{-} \cup \mathcal{E}^{+}\right)\right), \mathfrak{q}, \mathfrak{r} \in \mathfrak{P}$, such that $\mathbf{1}_{\mathfrak{q}} f \mathbf{1}_{\mathfrak{r}} \neq 0$, and for $U \in \mathcal{V}(\mathfrak{q})$, $W \in \mathcal{V}(\mathfrak{r})$, there exists a path $b$ in the labelled directed graph $G(\mathcal{V}, \Sigma, \lambda)$ from $U$ to $W$ such that $\lambda(b)=f$.

A finite labelled directed graph $G(\mathcal{V}, \Sigma, \lambda)$ that satisfies Conditions (G1)-(G5) gives rise to a subshift $X(G(\mathcal{V}, \Sigma, \lambda))$ that has as its language of admissible words the set of finite paths $b$ in the graph $G(\mathcal{V}, \Sigma, \lambda)$ such that $\lambda(b) \neq 0$. We say that $X(G(\mathcal{V}, \Sigma, \lambda))$ is presented by $G(\mathcal{V}, \Sigma, \lambda)$ or that $G(\mathcal{V}, \Sigma, \lambda)$ is an $\mathcal{S}\left(G_{\mathcal{R}}\left(\mathfrak{P}, \mathcal{E}^{-} \cup \mathcal{E}^{+}\right)\right)$-presentation of $X(G(\mathcal{V}, \Sigma, \lambda))$. From an $\mathcal{R}$-graph $G_{\mathcal{R}}\left(\mathfrak{P}, \mathcal{E}^{-} \cup \mathcal{E}^{+}\right)$, by using the identity map on the edge set $\mathcal{E}^{-} \cup \mathcal{E}^{+}$as label map, one obtains a particular case of an $\mathcal{S}\left(G_{\mathcal{R}}\left(\mathfrak{P}, \mathcal{E}^{-} \cup \mathcal{E}^{+}\right)\right)$presentation of a subshift $X\left(G\left(\mathfrak{P}, \mathcal{E}^{-} \cup \mathcal{E}^{+}, \mathrm{id}_{\mathcal{E}^{-} \cup \mathcal{E}^{+}}\right)\right)$that we call the $\mathcal{R}$-graph shift of 
$G_{\mathcal{R}}\left(\mathfrak{P}, \mathcal{E}^{-} \cup \mathcal{E}^{+}\right)$. In the case of the graph inverse semigroups $\mathcal{S}(G(\mathfrak{P}, \mathcal{E}))$ of strongly connected finite directed graphs $G(\mathfrak{P}, \mathcal{E})$, the subshifts $X(G(\mathfrak{P}, \mathcal{E}))$ are the Markov-Dyck shifts $[\mathbf{M}]$. The Dyck shifts $D_{N}$ can be obtained in this way from the one-vertex directed graph with $N>1$ loops. Also, the Markov-Motzkin shifts [KM1] of strongly connected finite directed graphs $G(\mathfrak{P}, \mathcal{E})$ have $\mathcal{S}(G(\mathfrak{P}, \mathcal{E})$ )-presentations.

Béal, Blockelet and Dima [BBD1, BBD2] have introduced the notions of a Dyck automaton and of a sofic Dyck shift. Strengthening the Condition (G1) to

$$
\lambda(\sigma) \in \mathcal{E}^{-} \cup\left\{\mathbf{1}_{\mathfrak{p}}: \mathfrak{p} \in \mathfrak{P}\right\} \cup \mathcal{E}^{+},
$$

one obtains labelled directed graphs $G(\mathcal{V}, \Sigma, \lambda)$, that are Dyck automata, and the subshifts $X(G(\mathcal{V}, \Sigma, \lambda))$ that arise from $\mathcal{S}\left(G\left(\mathfrak{P}, \mathcal{E}^{-} \cup \mathcal{E}^{+}\right)\right)$-presentations $G(\mathcal{V}, \Sigma, \lambda)$ are sofic Dyck shifts. The alphabet of a sofic Dyck shift is partitioned into a set of call symbols, a set of internal symbols and a set of return symbols. Under the assumption (1.1), the corresponding partition of the alphabet of the subshift $X(G(\mathcal{V}, \Sigma, \lambda))$ that is given by an $\mathcal{S}\left(G\left(\mathfrak{P}, \mathcal{E}^{-} \cup \mathcal{E}^{+}\right)\right)$-presentation $G(\mathcal{V}, \Sigma, \lambda)$ is the partition of its alphabet into the sets $\mathcal{E}^{-},\left\{\mathbf{1}_{\mathfrak{p}}, \mathfrak{p} \in \mathfrak{P}\right\}, \mathcal{E}^{+}$. The set of matched edges that appears in the construction of a Dyck automaton is provided by the relation $\mathcal{R}$. The $\mathcal{R}$-graph shifts are finite-type Dyck shifts in the sense of [BBD3].

Given finite sets $\mathcal{E}^{-}$and $\mathcal{E}^{+}$and a relation $\mathcal{R} \subset \mathcal{E}^{-} \times \mathcal{E}^{+}$, we set

$$
\mathcal{E}^{-}(\mathcal{R})=\left\{e^{-} \in \mathcal{E}^{-}:\left\{e^{-}\right\} \times \mathcal{E}^{+} \subset \mathcal{R}\right\}, \quad \mathcal{E}^{+}(\mathcal{R})=\left\{e^{+} \in \mathcal{E}^{+}: \mathcal{E}^{-} \times\left\{e^{+}\right\} \subset \mathcal{R}\right\} .
$$

For a partitioned directed graph $G\left(\mathfrak{P}, \mathcal{E}^{-} \cup \mathcal{E}^{+}\right)$, denote by $\mathfrak{P}^{(1)}$ the set of vertices in $\mathfrak{P}$ that have a single predecessor vertex in $G\left(\mathfrak{P}, \mathcal{E}^{-}\right)$or, equivalently, that have a single successor vertex in $G\left(\mathfrak{P}, \mathcal{E}^{+}\right)$. For $\mathfrak{p} \in \mathfrak{P}^{(1)}$ the predecessor vertex of $\mathfrak{p}$ in $G\left(\mathfrak{P}, \mathcal{E}^{-}\right)$, which is identical to the successor vertex of $\mathfrak{p}$ in $G\left(\mathfrak{P}, \mathcal{E}^{+}\right)$, is denoted by $\eta(\mathfrak{p})$. For an $\mathcal{R}$-graph $G_{\mathcal{R}}\left(\mathfrak{P}, \mathcal{E}^{-} \cup \mathcal{E}^{+}\right)$, we set

$$
\mathcal{E}_{\mathcal{R}}^{-}=\bigcup_{\mathfrak{p} \in \mathfrak{P}^{(1)}} \mathcal{E}^{-}(\mathcal{R}(\eta(\mathfrak{p}), \mathfrak{p})), \quad \mathcal{E}_{\mathcal{R}}^{+}=\bigcup_{\mathfrak{p} \in \mathfrak{P}^{(1)}} \mathcal{E}^{+}(\mathcal{R}(\eta(\mathfrak{p}), \mathfrak{p})),
$$

and

$$
\mathfrak{P}_{\mathcal{R}}^{(1)}=\left\{\mathfrak{p} \in \mathfrak{P}^{(1)}: \mathcal{R}(\eta(\mathfrak{p}), \mathfrak{p})=\mathcal{E}^{-}(\eta(\mathfrak{p}), \mathfrak{p}) \times \mathcal{E}^{+}(\eta(\mathfrak{p}), \mathfrak{p})\right\} .
$$

The subshift $X(G(\mathcal{V}, \Sigma, \lambda))$ that is given by the $\mathcal{S}\left(G_{\mathcal{R}}\left(\mathfrak{P}, \mathcal{E}^{-} \cup \mathcal{E}^{+}\right)\right)$-presentation $G(\mathcal{V}, \Sigma, \lambda)$ is a Markov shift if and only if $\mathfrak{P}=\mathfrak{P}_{\mathcal{R}}^{(1)}$. We formulate three Conditions (I), (II) and (III) on $\mathcal{R}$-graphs $G_{\mathcal{R}}\left(\mathfrak{P}, \mathcal{E}^{-} \cup \mathcal{E}^{+}\right)$such that

$$
\mathfrak{P} \backslash \mathfrak{P}_{\mathcal{R}}^{(1)} \neq \varnothing .
$$

Condition (II) comes in two parts (II-) and (II+) that are symmetric to one another.

(I) $\quad$ For $\mathfrak{p} \in \mathfrak{P}^{(1)} \backslash \mathfrak{P}_{\mathcal{R}}^{(1)}, \mathcal{E}^{-}(\mathcal{R}(\eta(\mathfrak{p}), \mathfrak{p}))=\emptyset$ or $\mathcal{E}^{+}(\mathcal{R}(\eta(\mathfrak{p}), \mathfrak{p}))=\emptyset$.

(II-) There is no cycle in the directed graph $G\left(\mathfrak{P}^{(1)}, \mathcal{E}_{\mathcal{R}}^{-}\right)$.

(II+) There is no cycle in the directed graph $G\left(\mathfrak{P}^{(1)}, \mathcal{E}_{\mathcal{R}}^{+}\right)$.

(III) For $\mathfrak{q}(-), \mathfrak{q}(+) \in \mathfrak{P}^{(1)}, \mathfrak{q}(-) \neq \mathfrak{q}(+)$, there does not simultaneously exist a path in $G\left(\mathfrak{P}^{(1)}, \mathcal{E}_{\mathcal{R}}^{+}\right)$from $\mathfrak{q}(-)$ to $\mathfrak{q}(+)$ and a path in $G\left(\mathfrak{P}^{(1)}, \mathcal{E}_{\mathcal{R}}^{-}\right)$from $\mathfrak{q}(-)$ to $\mathfrak{q}(+)$. 
We show in $\S 2$ that the subshift $X(G(\mathcal{V}, \Sigma, \lambda))$ that is given by an $\mathcal{S}\left(G_{\mathcal{R}}\left(\mathfrak{P}, \mathcal{E}^{-} \cup\right.\right.$ $\left.\mathcal{E}^{+}\right)$)-presentation $G(\mathcal{V}, \Sigma, \lambda)$ has Property $(A)$ if and only if the $\mathcal{R}$-graph $G_{\mathcal{R}}\left(\mathfrak{P}, \mathcal{E}^{-} \cup\right.$ $\left.\mathcal{E}^{+}\right)$satisfies Conditions (I), (II) and (III). In particular, the $\mathcal{R}$-graph shifts $X\left(G_{\mathcal{R}}\left(\mathfrak{P}, \mathcal{E}^{-} \cup\right.\right.$ $\left.\mathcal{E}^{+}\right)$) have Property $(A)$ if and only if the $\mathcal{R}$-graph $G_{\mathcal{R}}\left(\mathfrak{P}, \mathcal{E}^{-} \cup \mathcal{E}^{+}\right)$satisfies Conditions (I), (II) and (III). This implies that Markov-Dyck shifts of strongly connected finite directed graphs have Property $(A)$. Also, the Markov-Motzkin shifts of strongly connected finite directed graphs have Property $(A)$. Property $(A)$, and the semigroup $\mathcal{S}(X)$ of subshifts $X$ with Property $(A)$, in particular of $\mathcal{R}$-graph shifts, are invariants of flow equivalence (see [CS, §9] and [Kr3]).

In $\S 3$, we describe how one can obtain from an $\mathcal{R}$-graph $G_{\mathcal{R}}\left(\mathfrak{P}, \mathcal{E}^{-} \cup \mathcal{E}^{+}\right)$such that $\mathfrak{P} \backslash \mathfrak{P}_{\mathcal{R}}^{(1)} \neq \emptyset$ that satisfies Conditions (I), (II) and (III), an $\mathcal{R}$-graph $G_{\widehat{\mathcal{R}}}\left(\widehat{\mathfrak{P}}, \widehat{\mathcal{E}}^{-} \cup \widehat{\mathcal{E}}^{+}\right)$ such that one has for the subshifts $X(G(\mathcal{V}, \Sigma, \lambda))$ that are given by an $\mathcal{S}\left(G_{\mathcal{R}}\left(\mathfrak{P}, \mathcal{E}^{-} \cup\right.\right.$ $\left.\mathcal{E}^{+}\right)$)-presentation $G(\mathcal{V}, \Sigma, \lambda)$, that

$$
\mathcal{S}(X(G(\mathcal{V}, \Sigma, \lambda)))=\mathcal{S}\left(G_{\widehat{\mathcal{R}}}\left(\widehat{\mathfrak{P}}, \widehat{\mathcal{E}}^{-} \cup \widehat{\mathcal{E}}^{+}\right)\right) .
$$

To obtain the $\mathcal{R}$-graph $G_{\widehat{\mathcal{R}}}\left(\widehat{\mathfrak{P}}, \widehat{\mathcal{E}}^{-} \cup \widehat{\mathcal{E}}^{+}\right)$, we apply a procedure that extends a procedure for Markov-Dyck shifts that was described in [HK2] and [KM2].

In $\S 4$, we consider examples. We characterize by invariants of topological conjugacy the $\mathcal{R}$-graph shifts of one-vertex $\mathcal{R}$-graphs. We show how the isomorphism class of a onevertex $\mathcal{R}$-graph can be recovered from the topological conjugacy class of its $\mathcal{R}$-graph shift. For certain $\mathcal{R}$-graph semigroups of a one-vertex graph, see [HK1, §4].

We also introduce a family $\mathbf{F}$ of two-vertex $\mathcal{R}$-graphs. We characterize by invariants of topological conjugacy the $\mathcal{R}$-graph shifts of the $\mathcal{R}$-graphs in $\mathbf{F}$ and we show how the isomorphism class of an $\mathcal{R}$-graph in $\mathbf{F}$ can be recovered from the topological conjugacy class of its $\mathcal{R}$-graph shift. For other results on the reconstruction of a directed graph from its Markov-Dyck shift, see [KM1, §3] and [HK2].

\section{2. $\mathcal{S}\left(G_{\mathcal{R}}\left(\mathfrak{P}, \mathcal{E}^{-} \cup \mathcal{E}^{+}\right)\right)$-presentations of subshifts}

We consider an $\mathcal{R}$-graph

$$
G_{\mathcal{R}}=G_{\mathcal{R}}\left(\mathfrak{P}, \mathcal{E}^{-} \cup \mathcal{E}^{+}\right) .
$$

The symbol $\ell$ denotes the length of a directed path. There is the one-to-one correspondence between the non-empty directed paths in the directed graph $G\left(\mathfrak{P}, \mathcal{E}^{-}\right)\left(G\left(\mathfrak{P}, \mathcal{E}^{+}\right)\right)$and the elements of $\mathcal{S}^{-}\left(G_{\mathcal{R}}\right)\left(\mathcal{S}^{+}\left(G_{\mathcal{R}}\right)\right)$. We will use the same symbol to denote a nonempty directed path in $G\left(\mathfrak{P}, \mathcal{E}^{-}\right)\left(G\left(\mathfrak{P}, \mathcal{E}^{+}\right)\right)$and the corresponding element of $\mathcal{S}^{-}\left(G_{\mathcal{R}}\right)$ $\left(\mathcal{S}^{+}\left(G_{\mathcal{R}}\right)\right.$ ) (as we have already done for the edges in $\left.\mathcal{E}^{-} \cup \mathcal{E}^{+}\right)$. It will be clear from the context which one is meant. For the elements of $\mathcal{S}^{-}\left(G_{\mathcal{R}}\right)\left(\mathcal{S}^{+}\left(G_{\mathcal{R}}\right)\right)$, the notation $\ell, s, t$ is also used. We set $\ell\left(\mathbf{1}_{\mathfrak{p}}\right)=0, \mathfrak{p} \in \mathfrak{P}$. An element $g$ of $\mathcal{S}\left(G_{\mathcal{R}}\right) \backslash\{0\}$ determines uniquely

$$
\mathfrak{q}(g) \in \mathfrak{P}, \quad u^{+}(g) \in\left\{\mathbf{1}_{\mathfrak{q}(g)}\right\} \cup \mathcal{S}^{+}\left(G_{\mathcal{R}}\right), u^{-}(g) \in\left\{\mathbf{1}_{\mathfrak{q}(g)}\right\} \cup \mathcal{S}^{-}\left(G_{\mathcal{R}}\right),
$$

such that its normal form is given by

$$
g=u^{+}(g) \mathbf{1}_{\mathfrak{q}(g)} u^{-}(g) .
$$

We write the normal forms of elements $g^{-}$of $\mathcal{S}^{-}\left(G_{\mathcal{R}}\right)$ and of elements $g^{+}$of $\mathcal{S}^{+}\left(G_{\mathcal{R}}\right)$ as

$$
g^{-}=\mathbf{1}_{\mathfrak{q}\left(g^{-}\right)}\left(\prod_{1 \leq i(-) \leq \ell\left(g^{-}\right)} e_{i(-)}^{-}\left[g^{-}\right]\right), \quad g^{+}=\left(\prod_{1 \leq i(+) \leq \ell\left(g^{+}\right)} e_{i(+)}^{+}\left[g^{+}\right]\right) \mathbf{1}_{\mathfrak{q}\left(g^{+}\right)} .
$$


We denote the set of non-zero elements of the subsemigroup of $\mathcal{S}\left(G_{\mathcal{R}}\right)$ that is generated by $\mathcal{E}_{\mathcal{R}}^{-}\left(\mathcal{E}_{\mathcal{R}}^{+}\right)$by $\mathcal{S}_{\mathcal{R}}^{-}\left(G_{\mathcal{R}}\right)\left(\mathcal{S}_{\mathcal{R}}^{+}\left(G_{\mathcal{R}}\right)\right)$.

2.1. Context in $\mathcal{S}\left(G_{\mathcal{R}}\left(\mathfrak{P}, \mathcal{E}^{-} \cup \mathcal{E}^{+}\right)\right)$. In this subsection, we consider an $\mathcal{R}$-graph

$$
G_{\mathcal{R}}=G_{\mathcal{R}}\left(\mathfrak{P}, \mathcal{E}^{-} \cup \mathcal{E}^{+}\right)
$$

such that

$$
\mathfrak{P} \backslash \mathfrak{P}_{\mathcal{R}}^{(1)} \neq \varnothing,
$$

that satisfies Conditions (I), (II) and (III). For $f \in \mathcal{S}\left(G_{\mathcal{R}}\right)$, we set

$$
\begin{gathered}
\Gamma^{-}(f)=\left\{g \in \mathcal{S}\left(G_{\mathcal{R}}\right): g f \neq 0\right\}, \quad \Gamma^{+}(f)=\left\{g \in \mathcal{S}\left(G_{\mathcal{R}}\right): f g \neq 0\right\}, \\
\Gamma(f)=\left\{(g(-), g(+)) \in \mathcal{S}\left(G_{\mathcal{R}}\right)^{2}: g(-) f g(+) \neq 0\right\},
\end{gathered}
$$

and we refer to $\Gamma(f)$ as the context of $f$.

We denote for $\mathfrak{q}, \mathfrak{r} \in \mathfrak{P}$ by $\ell_{-}(\mathfrak{q}, \mathfrak{r})\left(\ell_{+}(\mathfrak{q}, \mathfrak{r})\right)$ the length of a path in $G\left(\mathfrak{P}, \mathcal{E}_{\mathcal{R}}^{-}\right)$ $\left(G\left(\mathfrak{P}, \mathcal{E}_{\mathcal{R}}^{+}\right)\right)$from $\mathfrak{q}$ to $\mathfrak{r}$, provided such a path exists. By Condition (II), this notation is meaningful.

We denote for $\mathfrak{q} \in \mathfrak{P}^{(1)}$ by $D_{+}(\mathfrak{q})$ the maximal length of a path in $G\left(\mathfrak{P}, \mathcal{E}_{\mathcal{R}}^{+}\right)$that leaves $\mathfrak{q}$, and by $D_{-}(\mathfrak{q})$ the maximal length of a path in $G\left(\mathfrak{P}, \mathcal{E}_{\mathcal{R}}^{-}\right)$that enters $\mathfrak{q}$. We also set

$$
D_{\circ}(\mathfrak{q})= \begin{cases}\min \left\{D_{+}(\mathfrak{q}), D_{-}(\mathfrak{q})\right\} & \text { if } \mathfrak{q} \in \mathfrak{P}^{(1)}, \\ 0 & \text { if } \mathfrak{q} \in \mathfrak{P} \backslash \mathfrak{P}^{(1)}\end{cases}
$$

We set inductively

$$
\eta^{k}(\mathfrak{q})=\eta\left(\eta^{k-1}(\mathfrak{q})\right), \quad 1<k<\max \left\{D_{-}(\mathfrak{q}), D_{+}(\mathfrak{q})\right\}, \quad \mathfrak{q} \in \mathfrak{P}^{(1)} .
$$

We remark that a path $b$ in $G\left(\mathfrak{P}, \mathcal{E}_{\mathcal{R}}^{+}\right)$that starts at $\mathfrak{q} \in \mathfrak{P}^{(1)}$ and that has length less than or equal to $D_{\circ}(\mathfrak{q})$ traverses the vertices $\eta^{k}(q), 1 \leq k<\ell(b)$, before entering its target vertex. Similarly, a path $b$ in $G\left(\mathfrak{P}, \mathcal{E}_{\mathcal{R}}^{-}\right)$that enters $\mathfrak{q} \in \mathfrak{P}^{(1)}$ and that has length less than or equal to $D_{\circ}(\mathfrak{q})$ traverses the vertices $\eta^{k}(q), \ell(b)>k \geq 1$, after leaving its source vertex.

For $\mathfrak{q} \in \mathfrak{P}^{(1)}$, we set $\eta^{0}(\mathfrak{q})=\mathfrak{q}$ and

$$
\mathfrak{R}_{-}(\mathfrak{q})=\left\{\eta^{k}(\mathfrak{q}): 0 \leq k \leq D_{-}(\mathfrak{q})\right\}, \quad \mathfrak{R}_{+}(\mathfrak{q})=\left\{\eta^{k}(\mathfrak{q}): 0 \leq k \leq D_{+}(\mathfrak{q})\right\},
$$

and, for $\mathfrak{q}(-), \mathfrak{q}(+) \in \mathfrak{P}^{(1)}$, such that

$$
\mathfrak{q}(-) \neq \mathfrak{q}(+), \quad \mathfrak{R}_{+}(\mathfrak{q}(-)) \cap \mathfrak{R}_{-}(\mathfrak{q}(+)) \neq \emptyset,
$$

we denote by $H_{+}(\mathfrak{q}(-), \mathfrak{q}(+))\left(H_{-}(\mathfrak{q}(-), \mathfrak{q}(+))\right)$ the minimal length of a path in $G\left(\mathfrak{P}, \mathcal{E}_{\mathcal{R}}^{+}\right)\left(G\left(\mathfrak{P}, \mathcal{E}_{\mathcal{R}}^{-}\right)\right)$that has $\mathfrak{q}(-)(\mathfrak{q}(+))$ as source (target) vertex and a vertex in $\mathfrak{R}_{-}(\mathfrak{q}(+))\left(\Re_{+}(\mathfrak{q}(-))\right)$ as target (source) vertex.

LEMMA 2.1. For $\mathfrak{q}(-), \mathfrak{q}(+) \in \mathfrak{P}^{(1)}$ such that

$$
\mathfrak{q}(-) \neq \mathfrak{q}(+), \quad \mathfrak{R}_{+}(\mathfrak{q}(-)) \cap \mathfrak{R}_{-}(\mathfrak{q}(+)) \neq \emptyset,
$$

one has that

$$
\eta^{H_{+}(\mathfrak{q}(-), \mathfrak{q}(+))}(\mathfrak{q}(-))=\eta^{H_{-}(\mathfrak{q}(-), \mathfrak{q}(+))}(\mathfrak{q}(+)) .
$$


Proof. The inequality

$$
\eta^{H_{+}(\mathfrak{q}(-), \mathfrak{q}(+))}(\mathfrak{q}(-)) \neq \eta^{H_{-}(\mathfrak{q}(-), \mathfrak{q}(+))}(\mathfrak{q}(+))
$$

would imply that one has $\widetilde{H}_{+}, \widetilde{H}_{-} \in \mathbb{N}$ such that

$$
\begin{array}{ll}
\eta^{H_{+}(\mathfrak{q}(-), \mathfrak{q}(+))}(\mathfrak{q}(-))=\eta^{\widetilde{H}_{-}}(\mathfrak{q}(+)), & \widetilde{H}_{-}>H_{-}(\mathfrak{q}(-), \mathfrak{q}(+)), \\
\eta^{H_{-}(\mathfrak{q}(-), \mathfrak{q}(+))}(\mathfrak{q}(+))=\eta^{\widetilde{H}_{+}}(\mathfrak{q}(-)), & \widetilde{H}_{+}>H_{+}(\mathfrak{q}(-), \mathfrak{q}(+)) .
\end{array}
$$

It follows then from the irreducibility assumption on $G(\mathfrak{P}, \mathcal{E})$ that

$$
\begin{aligned}
\mathfrak{P}=\left\{\eta^{k}(\mathfrak{q}(-)): H_{+}(\mathfrak{q}(-), \mathfrak{q}(+)) \leq k<\widetilde{H}_{+}\right\} \\
\cup\left\{\eta^{k}(\mathfrak{q}(+)): H_{-}(\mathfrak{q}(-), \mathfrak{q}(+)) \leq k<\widetilde{H}_{-}\right\} .
\end{aligned}
$$

Condition (III) leads to contradictions: if there is no path in $G\left(\mathfrak{P}, \mathcal{E}_{\mathcal{R}}^{+}\right)$from $q(-)$ to $q(+)$, then

$$
q(+) \notin\left\{\eta^{k}(\mathfrak{q}(-)): H_{+}(\mathfrak{q}(-), \mathfrak{q}(+)) \leq k<\widetilde{H}_{+}\right\},
$$

and, by Condition (II-),

$$
q(+) \notin\left\{\eta^{k}(\mathfrak{q}(+)): H_{-}(\mathfrak{q}(-), \mathfrak{q}(+)) \leq k<\widetilde{H}_{-}\right\},
$$

and, if there is no path in $G\left(\mathfrak{P}, \mathcal{E}_{\mathcal{R}}^{-}\right)$from $q(-)$ to $q(+)$, then

$$
q(-) \notin\left\{\eta^{k}(\mathfrak{q}(+)): H_{-}(\mathfrak{q}(-), \mathfrak{q}(+)) \leq k<\widetilde{H}_{-}\right\},
$$

and, by Condition (II+),

$$
q(-) \notin\left\{\eta^{k}(\mathfrak{q}(-)): H_{+}(\mathfrak{q}(-), \mathfrak{q}(+)) \leq k<\widetilde{H}_{+}\right\} .
$$

We denote the vertex that appears in $(2.2)$ by $\mathfrak{p}(\mathfrak{q}(-), \mathfrak{q}(+))$.

LEMMA A. Let $\mathfrak{q} \in \mathfrak{P}^{(1)}$. For

$$
f^{+} \in\left\{\mathbf{1}_{\mathfrak{q}}\right\} \cup \mathcal{S}_{\mathcal{R}}^{+}\left(G_{\mathcal{R}}\right), \quad f^{-} \in\left\{\mathbf{1}_{\mathfrak{q}}\right\} \cup \mathcal{S}_{\mathcal{R}}^{-}\left(G_{\mathcal{R}}\right),
$$

such that

$$
s\left(f^{+}\right)=\mathbf{1}_{\mathfrak{q}}=t\left(f^{-}\right), \quad t\left(f^{+}\right)=s\left(f^{-}\right),
$$

all elements of $\mathcal{S}\left(G_{\mathcal{R}}\right)$ of the form $f^{+} f^{-}$have the same context.

Proof. One notes that

$$
0 \leq \ell\left(f^{+}\right)=\ell\left(f^{-}\right) \leq D_{\circ}(\mathfrak{q})
$$

Set

$$
\begin{aligned}
& \Gamma_{\circ}^{-}(\mathfrak{q})=\left\{g(-) \in \Gamma^{-}(\mathfrak{q}): \ell\left(u^{-}(g(-)) \leq D_{\circ}(\mathfrak{q})\right\},\right. \\
& \Gamma_{\circ}^{+}(\mathfrak{q})=\left\{g(+) \in \Gamma^{+}(\mathfrak{q}): \ell\left(u^{+}(g(+)) \leq D_{\circ}(\mathfrak{q})\right\},\right. \\
& \Gamma_{\circ}(\mathfrak{q})=\left\{(g(-), g(+)) \in\left(\Gamma^{-}(\mathfrak{q}) \backslash \Gamma_{\circ}^{-}(\mathfrak{q})\right) \times\left(\Gamma^{+}(\mathfrak{q}) \backslash \Gamma_{\circ}^{+}(\mathfrak{q})\right):\right. \\
& \left(\prod_{1 \leq i(-)<\ell\left(u^{-}(g(-))\right)-D_{\circ}(\mathfrak{q})} e_{i(-)}^{-}\left[u^{-}(g(-))\right]\right) \mathbf{1}_{\eta D_{\circ}(\mathfrak{q})(\mathfrak{q})} \\
& \left.\times\left(\prod_{\ell\left(u^{+}(g(+))-D_{\circ}(\mathfrak{q})<i(+) \leq \ell\left(u^{+}(g(+))\right.\right.} e_{i(+)}^{+}\left[u^{+}(g(+))\right]\right) \neq 0\right\} .
\end{aligned}
$$


By Condition (II), one has for $f^{+}, f^{-}$as in (2.3) and (2.4),

$$
\Gamma\left(f^{+} f^{-}\right)=\left(\Gamma_{\circ}^{-}(\mathfrak{q}) \times \Gamma^{+}(\mathfrak{q})\right) \cup\left(\Gamma^{-}(\mathfrak{q}) \times \Gamma_{\circ}^{+}(\mathfrak{q})\right) \cup \Gamma_{\circ}(\mathfrak{q}) .
$$

LEMMA B. Let $\mathfrak{q}(-), \mathfrak{q}(+) \in \mathfrak{P}^{(1)}$ be such that

$$
\mathfrak{q}(-) \neq \mathfrak{q}(+) .
$$

$(B-)$ Let there exist a path in $S_{\mathcal{R}}^{+}\left(G_{\mathcal{R}}\right)$ from $\mathfrak{q}(-)$ to $\mathfrak{q}(+)$. Then, for

$$
h^{+} \in \mathcal{S}_{\mathcal{R}}^{+}\left(G_{\mathcal{R}}\right), \quad f^{+} \in\left\{\mathbf{1}_{\mathfrak{q}(+)}\right\} \cup \mathcal{S}_{\mathcal{R}}^{+}\left(G_{\mathcal{R}}\right), \quad f^{-} \in\left\{\mathbf{1}_{\mathfrak{q}(+)}\right\} \cup \mathcal{S}_{\mathcal{R}}^{-}\left(G_{\mathcal{R}}\right),
$$

such that

$$
\begin{gathered}
s\left(h^{+}\right)=\mathbf{1}_{\mathfrak{q}(-)}, \quad t\left(h^{+}\right)=\mathbf{1}_{\mathfrak{q}(+)}, \\
s\left(f^{+}\right)=\mathbf{1}_{\mathfrak{q}(+)}, \quad t\left(f^{+}\right)=s\left(f^{-}\right), \quad t\left(f^{-}\right)=\mathbf{1}_{\mathfrak{q}(+)},
\end{gathered}
$$

all elements of $\mathcal{S}\left(G_{\mathcal{R}}\right)$ of the form $h^{+} f^{+} f^{-}$have the same context. $(B+)$ Let there exist a path in $S_{\mathcal{R}}^{-}\left(G_{\mathcal{R}}\right)$ from $\mathfrak{q}(+)$ to $\mathfrak{q}(-)$. Then, for

$$
f^{+} \in\left\{\mathbf{1}_{\mathfrak{q}(-)}\right\} \cup \mathcal{S}_{\mathcal{R}}^{+}\left(G_{\mathcal{R}}\right), \quad f^{-} \in\left\{\mathbf{1}_{\mathfrak{q}(-)}\right\} \cup \mathcal{S}_{\mathcal{R}}^{-}\left(G_{\mathcal{R}}\right), \quad h^{-} \in \mathcal{S}_{\mathcal{R}}^{-}\left(G_{\mathcal{R}}\right),
$$

such that

$$
\begin{gathered}
s\left(h^{-}\right)=\mathbf{1}_{\mathfrak{q}(-)}, \quad t\left(h^{-}\right)=\mathbf{1}_{\mathfrak{q}(+)}, \\
s\left(f^{+}\right)=\mathbf{1}_{\mathfrak{q}(-)}, \quad t\left(f^{+}\right)=s\left(f^{-}\right), \quad t\left(f^{-}\right)=\mathbf{1}_{\mathfrak{q}(-)},
\end{gathered}
$$

all elements of $\mathcal{S}\left(G_{\mathcal{R}}\right)$ of the form $f^{+} f^{-} h^{-}$have the same context.

Proof. We prove (B-). We note that

$$
0 \leq \ell\left(f^{+}\right)=\ell\left(f^{-}\right) \leq D_{\circ}(\mathfrak{q}(+)) .
$$

Set

$$
\begin{aligned}
& \Gamma_{\circ}^{-}(\mathfrak{q}(-), \mathfrak{q}(+))=\left\{g(-) \in \Gamma^{-}\left(\mathbf{1}_{\mathfrak{q}(-)}\right): \ell\left(u^{+}(g(-))\right) \leq \ell(\mathfrak{q}(-), \mathfrak{q}(+))+D_{\circ}(\mathfrak{q}(+))\right\}, \\
& \Gamma_{\circ}^{+}(\mathfrak{q}(+))=\left\{g(+) \in \Gamma^{+}\left(\mathbf{1}_{\mathfrak{q}(+)}\right): \ell\left(u^{-}(g(+))\right) \leq D_{\circ}(\mathfrak{q}(+))\right\}, \\
& \Gamma_{\circ}(\mathfrak{q}(-), \mathfrak{q}(+)) \\
& \quad=(g(-), g(+)) \in\left(\Gamma^{-}(\mathfrak{q}(-)) \backslash \Gamma_{\circ}^{-}(\mathfrak{q}(-), \mathfrak{q}(+)) \times\left(\Gamma^{+}(\mathfrak{q}(+)) \backslash \Gamma_{\circ}^{+}(\mathfrak{q}(+))\right):\right. \\
& \quad\left(\prod_{1 \leq i(-)<\ell\left(u^{-}(g(-))\right)-\ell_{+}(\mathfrak{q}(-), \mathfrak{q}(+))-D_{\circ}(\mathfrak{q}(+))} e_{i(-)}^{-}\left[u^{-}(g(-))\right]\right) \mathbf{1}_{\eta^{D}(\mathfrak{q}(+))}(\mathfrak{q}(+)) \\
& \quad \times\left(\prod_{D_{\circ}(\mathfrak{q}(+))<i(+) \leq \ell\left(u^{+}(g(+))\right.} e_{i(+)}^{+}\left[u^{+}(g(+))\right]\right) \neq 0 .
\end{aligned}
$$

By Condition (II), one has for $h^{+}, f^{+}, f^{-}$as in (2.5) and (2.6) that

$$
\begin{aligned}
\Gamma & \left(h^{+} f^{+} f^{-}\right) \\
\quad & \left(\Gamma_{\circ}^{-}(\mathfrak{q}(-), \mathfrak{q}(+)) \times \Gamma^{+}(\mathfrak{q}(+)) \cup\left(\Gamma^{-}(\mathfrak{q}(-)) \times \Gamma_{\circ}^{+}(\mathfrak{q}(+))\right) \cup \Gamma_{\circ}(\mathfrak{q}(-), q(+)) .\right.
\end{aligned}
$$

The proof of $(\mathrm{B}+)$ is symmetric. 
LeMma C. Let $\mathfrak{q}(-), \mathfrak{q}(+) \in \mathfrak{P}^{(1)}$ be such that

$$
\mathfrak{q}(-) \neq \mathfrak{q}(+)
$$

and

$$
\mathfrak{R}_{+}(\mathfrak{q}(-)) \cap \mathfrak{R}_{-}(\mathfrak{q}(+)) \neq \emptyset, \quad \mathfrak{p}(\mathfrak{q}(-), \mathfrak{q}(+)) \notin\{\mathfrak{q}(-), \mathfrak{q}(+)\} .
$$

Then, for

$$
h^{+}, f^{+} \in \mathcal{S}_{\mathcal{R}}^{+}\left(G_{\mathcal{R}}\right), \quad f^{-}, h^{-} \in \mathcal{S}_{\mathcal{R}}^{-}\left(G_{\mathcal{R}}\right)
$$

such that

$$
\begin{gathered}
s\left(h^{+}\right)=\mathbf{1}_{\mathfrak{q}(-)}, \quad t\left(h^{+}\right)=\mathbf{1}_{\mathfrak{p}(\mathfrak{q}(-), \mathfrak{q}(+)),} \\
s\left(f^{+}\right)=\mathbf{1}_{\mathfrak{p}(\mathfrak{q}(-), \mathfrak{q}(+)),} t\left(f^{+}\right)=s\left(f^{-}\right), \quad t\left(f^{-}\right)=\mathbf{1}_{\mathfrak{p}(\mathfrak{q}(-), \mathfrak{q}(+)),} \\
s\left(h^{-}\right)=\mathbf{1}_{\mathfrak{p}(\mathfrak{q}(-), \mathfrak{q}(+)),} t\left(h^{-}\right)=\mathbf{1}_{\mathfrak{q}(+)},
\end{gathered}
$$

all elements of $\mathcal{S}\left(G_{\mathcal{R}}\right)$ of the form $h^{+} f^{+} f^{-} h^{-}$have the same context.

Proof. One notes that

$$
0 \leq \ell\left(f^{+}\right)=\ell\left(f^{-}\right) \leq D_{\circ}(\mathfrak{p}(\mathfrak{q}(-), \mathfrak{q}(+)))
$$

Set

$$
\begin{aligned}
\Gamma_{\circ}^{-}(\mathfrak{q}(-), \mathfrak{q}(+)) & \\
= & \left\{g(-) \in \Gamma^{-}\left(\mathbf{1}_{\mathfrak{q}(-)}\right): \ell\left(u^{-}(g(-))\right) \leq H_{-}(\mathfrak{q}(-), \mathfrak{q}(+))+D_{\circ}(\mathfrak{p}(\mathfrak{q}(-), \mathfrak{q}(+)))\right\}, \\
\Gamma_{\circ}^{-}(\mathfrak{q}(-), \mathfrak{q}(+)) & \left\{g(+) \in \Gamma^{+}\left(\mathbf{1}_{\mathfrak{q}(+)}\right): \ell\left(u^{+}(g(+))\right) \leq H_{+}(\mathfrak{q}(-), \mathfrak{q}(+))+D_{\circ}(\mathfrak{p}(\mathfrak{q}(-), \mathfrak{q}(+)))\right\}, \\
= & \prod_{\circ}(\mathfrak{q}(-), \mathfrak{q}(+)) \\
= & \left\{( g ( - ) , g ( + ) ) \in \left(\Gamma^{-}\left(\mathbf{1}_{\mathfrak{q}(-)}\right) \backslash \Gamma_{\circ}^{-}(\mathfrak{q}(-), \mathfrak{q}(+)) \times\left(\Gamma^{+}\left(\mathbf{1}_{\mathfrak{q}(+)}\right) \backslash \Gamma_{\circ}^{+}(\mathfrak{q}(+), \mathfrak{q}(+))\right):\right.\right. \\
& \left(\begin{array}{l}
\left.e_{i(-)}^{-}\left[u^{-}(g(-))\right]\right) \\
1 \leq i(-)<\ell\left(u^{-}(g(-))\right)-H_{+}(\mathfrak{q}(-), \mathfrak{q}(+))-D_{\circ}(\mathfrak{p}(\mathfrak{q}(-), \mathfrak{q}(+))
\end{array}\right. \\
& \times \mathbf{1}_{\eta^{\circ}(\mathfrak{p}(\mathfrak{q}(-), \mathfrak{q}(+)))}(\mathfrak{p}(\mathfrak{q}(-), \mathfrak{q}(+)) \\
& \times\left(\begin{array}{l}
\prod_{\ell\left(u^{+}(g(+))\right)-H_{-}(\mathfrak{q}(-), \mathfrak{q}(+))-D_{\circ}\left(\mathfrak{p}(\mathfrak{q}(-))<i(+) \leq \ell\left(u^{+}(g(+))\right.\right.} \\
\left.\left.\left.\quad e_{i(+)}^{+}\left[u^{+}(g(+))\right)\right]\right) \neq 0\right\} .
\end{array}\right.
\end{aligned}
$$

By Condition (II), one has for $h^{+}, f^{+}, f^{-}, h^{-}$as in (2.7) and (2.8) that

$$
\begin{aligned}
\Gamma\left(h^{+} f^{+} f^{-} h^{-}\right)= & \left(\Gamma_{\circ}^{-}(\mathfrak{q}(-), \mathfrak{q}(+)) \times \Gamma^{+}\left(\mathbf{1}_{\mathfrak{q}(+)}\right) \cup\left(\Gamma^{-}\left(\mathbf{1}_{\mathfrak{q}(-)}\right)\right.\right. \\
& \left.\times \Gamma_{\circ}^{+}(\mathfrak{q}(-), \mathfrak{q}(+))\right) \cup \Gamma_{\circ}(\mathfrak{q}(-), q(+)) .
\end{aligned}
$$


2.2. Property $(A)$ and $\mathcal{R}$-graph shifts. We introduce notation and terminology for subshifts. The set of periodic points of a subshift $X$ we denote by $P(X)$. The smallest period of $p \in P(X)$ we denote by $\pi(p)$. We denote the language of admissible words of a subshift $X \subset \Sigma^{\mathbb{Z}}$ by $\mathcal{L}(X)$. The context of a word $b \in \mathcal{L}(X)$ is defined as the set

$$
\Gamma(b)=\left\{(c(-), c(+)) \in \mathcal{L}(X)^{2}: c(-) b c(+) \in \mathcal{L}(X)\right\} .
$$

Concerning the subshifts $X(G(\mathcal{V}, \Sigma, \lambda))$ that are given by $\mathcal{S}\left(G\left(\mathfrak{P}, \mathcal{E}^{-} \cup \mathcal{E}^{+}\right)\right)$presentations $G(\mathcal{V}, \Sigma, \lambda)$, we remark that

$$
\begin{gathered}
\Gamma(b)=\{(c(-), c(+)) \in \mathcal{L}(X(\mathcal{V}, \Sigma, \lambda)))^{2}: t(c(-))=s(b), t(b)=s(c(+)), \\
(\lambda(c(-)), \lambda(c(+))) \in \Gamma(\lambda(b))\}, \quad b \in \mathcal{L}(X(\mathcal{V}, \Sigma, \lambda)) .
\end{gathered}
$$

Given a subshift $X \subset \Sigma^{\mathbb{Z}}$, we set

$$
x_{[i, k]}=\left(x_{j}\right)_{i \leq j \leq k}, \quad x \in X, i, k \in \mathbb{Z}, i \leq k,
$$

and

$$
X_{[i, k]}=\left\{x_{[i, k]}: x \in X\right\}, \quad i, k \in \mathbb{Z}, i \leq k .
$$

We set

$$
\begin{gathered}
\Gamma(a)=\bigcup_{n, m \in \mathbb{N}}\left\{(b, c) \in X_{[i-n, i]} \times X_{[k, k+m]}:(b, a, c) \in X_{[i-n, k+m]}\right\}, \\
a \in X_{[i, k]}, i, k \in \mathbb{Z}, i \leq k,
\end{gathered}
$$

and call $\Gamma(a)$ the context of the block $a$. With the notation

$$
\Gamma_{n}^{-}(a)=\left\{c \in X_{[i-n, i)]}:(c, a) \in X_{[i-n, k]}\right\}, \quad n \in \mathbb{N}, a \in X_{[i, k]}, i, k \in \mathbb{Z}, i \leq k,
$$

we set

$$
\begin{gathered}
\omega^{+}(a)=\bigcup \begin{array}{c}
\bigcap_{m \in \mathbb{N}} \\
\bigcap_{n \in \mathbb{N}}
\end{array} \bigcap_{c \in \Gamma_{n}^{-}(a)}\left\{b \in X_{(k, k+m]}:(c, a, b) \in X_{[i-n, k+m]}\right\}, \\
a \in X_{[i, k]}, i, k \in \mathbb{Z}, i \leq k .
\end{gathered}
$$

Given a subshift $X \subset \Sigma^{\mathbb{Z}}$, we define a subshift of finite type (more precisely, an $n$-step Markov shift) $A_{n}^{+}(X)$ by

$$
A_{n}^{-}(X)=\bigcap_{i \in \mathbb{Z}}\left(\left\{x \in X: x_{i} \in \omega^{+}\left(x_{[i-n, i)}\right)\right\}\right), \quad n \in \mathbb{N},
$$

and we set

$$
A^{-}(X)=\bigcup_{n \in \mathbb{N}} A_{n}^{-}(X) .
$$

The symbol $A^{+}$has the time-symmetric meaning. We set

$$
A(X)=A^{-}(X) \cap A^{+}(X) .
$$

We recall from [Kr2] the definition of Property $(A)$. For $n \in \mathbb{N}$, a subshift $X \subset \Sigma^{\mathbb{Z}}$ has property $(a, n, H), H \in \mathbb{N}$, if, for $h, \widetilde{h} \geq 3 H$ and for $I_{-}, I_{+}, \widetilde{I}_{-}, \widetilde{I}_{+} \in \mathbb{Z}$, such that

$$
I_{+}-I_{-}, \tilde{I}_{+}-\tilde{I}_{-} \geq 3 H
$$


and for

$$
a \in A_{n}(X)_{\left(I_{-}, I_{+}\right]}, \quad \tilde{a} \in A_{n}(X)_{\left(\tilde{I}_{-}, \tilde{I}_{+}\right]},
$$

such that

$$
a_{\left(I_{-}, I_{-}+H\right]}=\tilde{a}_{\left(\widetilde{I}_{-}, \tilde{I}_{-}+H\right]}, \quad a_{\left(I_{+}-H, I_{+}\right]}=\tilde{a}_{\left(\widetilde{I}_{+}-H, \widetilde{I}_{+}\right]},
$$

one has that $a$ and $\tilde{a}$ have the same context. A subshift $X \subset \Sigma^{\mathbb{Z}}$ such that $A(X) \neq \emptyset$ has Property $(A)$ if there are $H_{n}, n \in \mathbb{N}$, such that $X$ has the properties $\left(a, n, H_{n}\right), n \in \mathbb{N}$. Subshifts of finite type have Property $(A)$.

THEOREM 2.2. Let

$$
G_{\mathcal{R}}=G_{\mathcal{R}}\left(\mathfrak{P}, \mathcal{E}^{-} \cup \mathcal{E}^{+}\right),
$$

be an $\mathcal{R}$-graph such that

$$
\mathfrak{P}^{(1)} \backslash \mathfrak{P}_{\mathcal{R}}^{(1)} \neq \varnothing .
$$

For a subshift $X(G(\mathcal{V}, \Sigma, \lambda))$ that is given by an $\mathcal{S}_{\mathcal{R}}\left(\mathfrak{P}, \mathcal{E}^{-} \cup \mathcal{E}^{+}\right)$-presentation $G(\mathcal{V}, \Sigma, \lambda)$ to have Property $(A)$, it is necessary and sufficient that $G_{\mathcal{R}}\left(\mathfrak{P}, \mathcal{E}^{-} \cup \mathcal{E}^{+}\right)$ satisfies Conditions (I), (II) and (III).

Proof. We prove necessity. We choose vertices $V_{\mathfrak{p}} \in \mathcal{V}(\mathfrak{p}), \mathfrak{p} \in \mathfrak{P}$, and simple cycles $c_{\mathfrak{p}}, \mathfrak{p} \in \mathfrak{P}$, in the graph $G(\mathcal{V}, \Sigma)$ such that

$$
s\left(c_{\mathfrak{p}}\right)=t\left(c_{\mathfrak{p}}\right)=V_{\mathfrak{p}}, \quad \lambda\left(c_{\mathfrak{p}}\right)=\mathbf{1}_{\mathfrak{p}}, \quad \mathfrak{p} \in \mathfrak{P} .
$$

For $k \in \mathbb{N}$, we denote by $c_{\mathfrak{p}}^{k}$ the cycle that traverses $k$ times the cycle $c_{\mathfrak{p}}$. Also, we choose for all $e^{-} \in \mathcal{E}^{-}$a path $a_{e^{-}}$in the graph $G(\mathcal{V}, \Sigma)$ such that

$$
s\left(a_{e^{-}}\right)=V_{s\left(e^{-}\right)}, \quad t\left(a_{e^{-}}\right)=V_{t\left(e^{-}\right)}, \quad \lambda\left(a_{e^{-}}\right)=e^{-},
$$

and we make similar choices for all $e^{+} \in \mathcal{E}^{+}$. For a path $f^{-}$in the graph $G\left(\mathfrak{P}, \mathcal{E}^{-}\right)$, we set

$$
a_{f^{-}}=\left(a_{e_{l}^{-}}\right)_{1 \leq l \leq \ell\left(f^{+}\right)}
$$

and we use similar notation for paths $f^{-}$in the graph $G\left(\mathfrak{P}, \mathcal{E}^{+}\right)$.

We set

$$
M=\max \left(\left\{\ell\left(a_{e^{-}}\right): e^{-} \in \mathcal{E}^{-}\right\} \cup\left\{\ell\left(c_{\mathfrak{p}}\right): \mathfrak{p} \in \mathfrak{P}\right\} \cup\left\{\ell\left(a_{e^{+}}\right): e^{+} \in \mathcal{E}^{+}\right\}\right) .
$$

One has that

$$
c_{\mathfrak{p}}^{k} \in \mathcal{L}\left(A_{M}(X(\mathcal{V}, \Sigma, \lambda))\right), \quad k \in \mathbb{N}, \mathfrak{p} \in \mathfrak{P} .
$$

(I) Assume that the $\mathcal{R}$-graph $\mathcal{G}_{\mathcal{R}}\left(\mathfrak{P}, \mathcal{E}^{-} \cup \mathcal{E}^{+}\right)$does not satisfy Condition (I). Under this assumption, we can choose a vertex $\mathfrak{p} \in \mathfrak{P}^{(1)} \backslash \mathfrak{P}_{\mathcal{R}}^{(1)}$ and edges

$$
e^{-} \in \mathcal{E}_{\mathcal{R}}^{-}(\eta(\mathfrak{p}), \mathfrak{p}), \quad e^{+} \in \mathcal{E}_{\mathcal{R}}^{+}(\eta(\mathfrak{p}), \mathfrak{p}),
$$

together with edges

$$
\tilde{e}^{-} \in \mathcal{E}^{-}(\eta(\mathfrak{p}), \mathfrak{p}), \quad \widetilde{e}^{+} \in \mathcal{E}^{+}(\eta(\mathfrak{p}), \mathfrak{p}),
$$

such that

$$
\left(\widetilde{e}^{-}, \widetilde{e}^{+}\right) \notin \mathcal{R}(\eta(\mathfrak{p}), \mathfrak{p}) .
$$


By construction,

$$
\left(c_{\mathfrak{p}}^{k}, a_{e^{+}}, a_{e^{-}}, c_{\mathfrak{p}}^{k}\right) \in \mathcal{L}\left(A_{M}(X(\mathcal{V}, \Sigma, \lambda))\right), \quad k \in \mathbb{N} .
$$

It follows from (2.10) that

$$
\left(a_{\widetilde{e}^{-}}, a_{\widetilde{e}^{+}}\right) \in \Gamma\left(c_{\mathfrak{p}}^{k}, a_{e^{+}}, a_{e^{-}}, c_{\mathfrak{p}}^{k}\right), \quad k \in \mathbb{N},
$$

and it follows from (2.11) that

$$
\left(a_{\widetilde{e}^{-}}, a_{\widetilde{e}^{+}}\right) \notin \Gamma\left(c_{\mathfrak{p}}^{2 k}\right), \quad k \in \mathbb{N} .
$$

We have shown that $X(G(\mathcal{V}, \Sigma, \lambda))$ does not have Property $(A)$.

(II) Assume that the $\mathcal{R}$-graph $G_{\mathcal{R}}\left(\mathfrak{P}, \mathcal{E}^{-} \cup \mathcal{E}^{+}\right)$does not satisfy Condition (II-). By (2.9), every cycle in $G\left(\mathfrak{P}, \mathcal{E}^{-}\right)$traverses at least one vertex in $\mathfrak{P}^{(1)} \backslash \mathfrak{P}_{\mathcal{R}}^{(1)}$. We can therefore choose a cycle

$$
f^{-}=\left(e_{l}^{-}\right)_{1 \leq l \leq \ell\left(f^{-}\right)}
$$

in the graph $G\left(\mathfrak{P}, \mathcal{E}_{\mathcal{R}}^{-}\right)$and a vertex $\mathfrak{p} \in \mathfrak{P}^{(1)} \backslash \mathfrak{P}_{\mathcal{R}}^{(1)}$ such that

$$
s\left(f^{-}\right)=\mathfrak{p}=t\left(f^{-}\right),
$$

together with edges

$$
\widetilde{e}^{-} \in \mathcal{E}_{\mathcal{R}}^{-}(\eta(\mathfrak{p}), \mathfrak{p}), \quad \widetilde{e}^{+} \in \mathcal{E}_{\mathcal{R}}^{+}(\eta(\mathfrak{p}), \mathfrak{p}),
$$

such that

$$
t\left(\widetilde{e}^{-}\right)=\mathfrak{p}=s\left(\widetilde{e}^{+}\right),
$$

and such that

$$
\left(\widetilde{e}^{-}, \widetilde{e}^{+}\right) \notin \mathcal{R}(\eta(\mathfrak{p}), \mathfrak{p}) .
$$

By construction,

$$
\left(c_{\mathfrak{p}}^{k}, a_{f^{-}}, c_{\mathfrak{p}}^{k}\right) \in \mathcal{L}\left(A_{M}(X(G(\mathcal{V}, \Sigma, \lambda))), \quad k \in \mathbb{N} .\right.
$$

It follows from (2.12) that

$$
\left(a_{\widetilde{e}^{-}}, a_{\widetilde{e}^{+}}\right) \in \Gamma\left(c_{\mathfrak{p}}^{k}, a_{f^{-}}, c_{\mathfrak{p}}^{k}\right), \quad k \in \mathbb{N},
$$

and it follows from (2.13) that

$$
\left(a_{\widetilde{e}^{-}}, a_{\widetilde{e}^{+}}\right) \notin \Gamma\left(c_{\mathfrak{p}}^{2 k}\right), \quad k \in \mathbb{N} .
$$

We have shown that $X(G(\mathcal{V}, \Sigma, \lambda))$ does not have Property $(A)$.

For Condition (II+) one has the symmetric argument.

(III) Assume that the $\mathcal{R}$-graph $G_{\mathcal{R}}\left(\mathfrak{P}, \mathcal{E}^{-} \cup \mathcal{E}^{+}\right)$does not satisfy Condition (III). Under this assumption, we can choose by (2.9) vertices $\mathfrak{q}(-), \mathfrak{q}(+) \in \mathfrak{P}^{(1)}$, together with a path $f^{+}=\left(e_{i(+)}^{+}\right)_{1 \leq i(+) \leq I(+)}$ from $\mathfrak{q}(-)$ to $\mathfrak{q}(+)$ in the graph $G\left(\mathfrak{P}^{(1)}, \mathcal{E}_{\mathcal{R}}^{+}\right)$and a path $f^{-}=$ $\left(e_{i(-)}^{-}\right)_{1 \leq i(-) \leq I(-)}$ from $\mathfrak{q}(-)$ to $\mathfrak{q}(+)$ in the graph $G\left(\mathfrak{P}^{(1)}, \mathcal{E}_{\mathcal{R}}^{-}\right)$, such that

$$
\left\{s\left(e_{i(+)}^{+}\right): 1 \leq i(+) \leq I(+)\right\} \backslash \mathfrak{P}_{\mathcal{R}}^{(1)} \neq \emptyset,
$$

or such that

$$
\left\{s\left(e_{i(-)}^{+}\right): 1 \leq i(-) \leq I(-)\right\} \backslash \mathfrak{P}_{\mathcal{R}}^{(1)} \neq \emptyset .
$$


Assume (2.14). Set

$$
g^{+}=\left(e_{i}^{+}\right)_{1 \leq i \leq D_{\circ}(\mathfrak{q}(-))}, \quad \mathfrak{p}=t\left(e_{D_{\circ}(\mathfrak{q}(-))}^{+}\right),
$$

and let $g^{-}$be a path in $G\left(\mathfrak{P}^{(1)}, \mathcal{E}_{\mathcal{R}}^{-}\right)$from $\mathfrak{p}$ to $\mathfrak{q}(-)$. Choose a path $\tilde{f}^{+}$in the graph $G\left(\mathfrak{P}, \mathcal{E}^{+}\right)$from $\mathfrak{q}(+)$ to $\mathfrak{q}(-)$ such that

$$
f^{-} \tilde{f}^{+}=\mathbf{1}_{\mathfrak{q}(-)} .
$$

Also, choose

$$
e^{-} \in \mathcal{E}^{-}(\eta(\mathfrak{p}), \mathfrak{p}), \quad e^{+} \in \mathcal{E}^{+}(\eta(\mathfrak{p}), \mathfrak{p}),
$$

such that

$$
\left(e^{-}, e^{+}\right) \notin \mathcal{R}(\eta(\mathfrak{p}), \mathfrak{p}) .
$$

By construction,

$$
\begin{array}{r}
\left(c_{\mathfrak{p}}^{k}, a_{g^{-}}, a_{f^{+}}, a_{\tilde{f}^{+}}, a_{g^{+}}, c_{\mathfrak{p}}^{k}\right),\left(c_{\mathfrak{p}}^{k}, a_{g^{-}}, a_{f^{-}}, a_{\tilde{f}^{+}}, a_{g^{+}}, c_{\mathfrak{p}}^{k}\right) \in \mathcal{L}\left(A_{M}(X(\mathcal{V}, \Sigma, \lambda))\right), \\
k \in \mathbb{N} .
\end{array}
$$

One has that

$$
\left(a_{e^{-}}, a_{e^{+}}\right) \in \Gamma\left(c_{\mathfrak{p}}^{k}, a_{g^{-}}, a_{f^{+}}, a_{\tilde{f}^{+}}, a_{g^{+}}, c_{\mathfrak{p}}^{k}\right), \quad k \in \mathbb{N},
$$

and, by (2.16), one has that

$$
\left(a_{e^{-}}, a_{e^{+}}\right) \notin \Gamma\left(c_{\mathfrak{p}}^{k}, a_{g^{-}}, a_{f^{-}}, a_{\tilde{f}^{+}}, a_{g^{+}}, c_{\mathfrak{p}}^{k}\right), \quad k \in \mathbb{N} .
$$

We have shown that $X(G(\mathcal{V}, \Sigma, \lambda))$ does not have Property $(A)$. Under the assumption (2.15) one has the symmetric argument.

We prove sufficiency. Let $n \in \mathbb{N}$ and let $a(-), a(+) \in \mathcal{L}(X(G(\mathcal{V}, \Sigma, \lambda))), \ell(a(-))=$ $\ell(a(+))=n$. For $m \in[1, n]$, denote by $a(-)_{[1, m]}\left(a(+)_{[m, n]}\right)$ the prefix (suffix) of length $m(n-m+1)$ of $a(-)(a(+))$. Set

$$
\begin{aligned}
& M(-)= \begin{cases}\max \left\{m \in[1, n]: \lambda\left(a(-)_{[1, m]}\right)=u^{+}(\lambda(a(-))\}\right. & \text { if } u^{+}\left(\lambda(a(-)) \in \mathcal{S}^{+}\left(G_{\mathcal{R}}\right),\right. \\
0 & \text { if } u^{+}\left(\lambda(a(-))=\mathbf{1}_{\mathfrak{q}(\lambda(a(-)),}\right.\end{cases} \\
& M(+)= \begin{cases}\max \left\{m \in[1, n]: \lambda\left(a(+)_{[n-m, n]}\right)=u^{-}(\lambda(a(-))\}\right. & \text { if } u^{-}\left(\lambda(a(+)) \in \mathcal{S}^{-}\left(G_{\mathcal{R}}\right),\right. \\
0 & \text { if } u^{-}\left(\lambda(a(+))=\mathbf{1}_{\mathfrak{q}(\lambda(a(+))} .\right.\end{cases}
\end{aligned}
$$

Let

$$
\begin{gathered}
K(-), K(+), \bar{K}(-), \bar{K}(+) \in \mathbb{Z}, \\
K(+)-K(-), \bar{K}(+)-\bar{K}(-)>2 n
\end{gathered}
$$

and let

$$
b \in A_{n}(X(G))_{[K(-), K(+)]}, \quad \bar{b} \in A_{n}(X(G))_{[\bar{K}(-), \bar{K}(+)]}
$$

be such that

$$
\begin{aligned}
& b_{[K(-), K(-)+n]}=\bar{b}_{[\bar{K}(-), \bar{K}(-)+n]}=a(-), \\
& b_{[K(+)-n, K(+)]}=\bar{b}_{[\bar{K}(+)-n, \bar{K}(+)]}=a(+) .
\end{aligned}
$$


Set

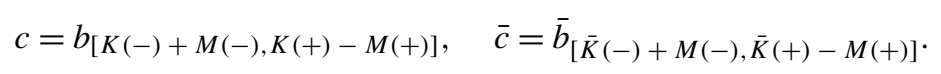

We consider the four cases $(\mathrm{A}),(\mathrm{B}-),(\mathrm{B}+)$ and $(\mathrm{C})$.

(A) In the case that $\mathfrak{q}(\lambda(a(-)))=\mathfrak{q}(a(-))$, set

$$
f^{+}=u^{+}(\lambda(c)), \quad f^{-}=u^{-}(\lambda(c)), \quad \bar{f}^{+}=\bar{u}^{+}(\lambda(\bar{c})), \quad \bar{f}^{-}=\bar{u}^{-}(\lambda(\bar{c})) .
$$

It follows from (2.17) that

By Lemma A,

$$
f^{+}, \bar{f}^{+} \in \mathcal{S}_{\mathcal{R}}^{+}\left(G_{\mathcal{R}}\right), \quad f^{-}, \bar{f}^{-} \in \mathcal{S}_{\mathcal{R}}^{-}\left(G_{\mathcal{R}}\right) .
$$

$$
\Gamma\left(f^{+} f^{-}\right)=\Gamma\left(\bar{f}^{+} \bar{f}^{-}\right) .
$$

(B-) In the case that

$$
\mathfrak{q}(\lambda(a(-))) \neq \mathfrak{q}(\lambda(a(+)), \quad \mathfrak{q}(\lambda(a(-)))=\mathfrak{p}(\mathfrak{q}(\lambda(a(-))), \mathfrak{q}(\lambda(a(+))),
$$

one has

$$
\ell\left(u^{+}(\lambda(c)), \ell\left(u^{+}(\lambda(c)) \geq \ell_{+}(\mathfrak{q}(\lambda(a(-)), \mathfrak{q}(\lambda(a(+))) .\right.\right.
$$

Let

$$
h^{+}, \quad f^{+}, f^{-}, \quad \bar{h}^{+}, \bar{f}^{+}, \bar{f}^{-},
$$

be given by

$$
\begin{gathered}
\ell\left(h^{+}\right)=\ell\left(\bar{h}^{+}\right)=\ell_{+}(\mathfrak{q}(\lambda(a(-)), \mathfrak{q}(\lambda(a(+)), \\
h^{+}=u^{+}(\lambda(c)) f^{+}, \quad \bar{h}^{+}=u^{+}(\lambda(\bar{c})) f^{+} .
\end{gathered}
$$

By (2.17),

$$
h^{+}, \bar{h}^{+}, f^{+}, \bar{f}^{+} \in \mathcal{S}_{\mathcal{R}}^{-}\left(G_{\mathcal{R}}\right), \quad f^{-}, \bar{f}^{-} \in \mathcal{S}_{\mathcal{R}}^{+}\left(G_{\mathcal{R}}\right) .
$$

By Lemma B(-),

$$
\Gamma\left(h^{+} f^{+} f^{-}\right)=\Gamma\left(\bar{h}^{+} \bar{f}^{+} \bar{f}^{-}\right) .
$$

The case $\mathrm{B}(+)$ is symmetric.

(C) In the case that

$$
\begin{aligned}
\mathfrak{q}(\lambda(a(-))) & \neq \mathfrak{q}(\lambda(a(+)), \\
\mathfrak{p}(\mathfrak{q}(\lambda(a(-))), \mathfrak{q}(\lambda(a(+)))) & \notin\{\mathfrak{q}(\lambda(a(-))), \mathfrak{q}(\lambda(a(+)))\},
\end{aligned}
$$

let

$$
h^{+} \bar{h}^{+}, f^{+}, \bar{f}^{+} \in \mathcal{S}_{\mathcal{R}}^{+}\left(G_{\mathcal{R}}\right), \quad f^{-}, \bar{f}^{-}, h^{-}, \bar{h}^{-} \in \mathcal{S}_{\mathcal{R}}^{-}\left(G_{\mathcal{R}}\right),
$$

be given by

$$
\begin{gathered}
\ell\left(h^{+}\right)=\ell\left(\bar{h}^{+}\right)=\ell_{+}(\mathfrak{q}(\lambda(a(-))), \mathfrak{p}(\mathfrak{q}(\lambda(a(-))), \mathfrak{q}(\lambda(a(+))))), \\
\ell\left(h^{-}\right)=\ell\left(\bar{h}^{-}\right)=\ell_{-}(\mathfrak{p}(\mathfrak{q}(\lambda(a(-)))), \mathfrak{q}(\lambda(a(+))), \mathfrak{q}(\lambda(a(+)))), \\
u^{+}(c)=h^{+} f^{+}, \quad u^{+}(\bar{c})=\bar{h}^{+} \bar{f}^{+}, \quad u^{-}(c)=h^{-} f^{-}, \quad u^{-}(\bar{c})=\bar{h}^{-} \bar{f}^{-} .
\end{gathered}
$$

By (2.17),

$$
h^{+}, \bar{h}^{+}, f^{+}, \bar{f}^{+} \in \mathcal{S}_{\mathcal{R}}^{-}\left(G_{\mathcal{R}}\right), \quad f^{-}, \bar{f}^{-}, h^{+}, \bar{h}^{+} \in \mathcal{S}_{\mathcal{R}}^{+}\left(G_{\mathcal{R}}\right) .
$$

By Lemma C,

$$
\Gamma\left(h^{+} f^{+} f^{-} h^{-}\right)=\Gamma\left(\bar{h}^{+} \bar{f}^{+} \bar{f}^{-} \bar{h}^{-}\right) .
$$

It follows from (2.18)-(2.20) that in all cases the context of $b$ is equal to the context of $\bar{b}$. It is shown that $X(G(\mathcal{V}, \Sigma, \lambda))$ has properties $a(n, n), n \in \mathbb{N}$. 
3. The $\mathcal{R}$-graph semigroup $\mathcal{S}(X(G(\mathcal{V}, \Sigma, \lambda)))$

Following the terminology that was introduced in $[\mathbf{H I}]$, we say, given an $\mathcal{R}$-graph $G_{\mathcal{R}}\left(\mathfrak{P}, \mathcal{E}^{-} \cup \mathcal{E}^{+}\right)$and a subshift $X(G(\mathcal{V}, \Sigma, \lambda))$ that is given by an $\mathcal{S}\left(G_{\mathcal{R}}\left(\mathfrak{P}, \mathcal{E}^{-}, \mathcal{E}^{+}\right)\right)$presentation $G(\mathcal{V}, \Sigma, \lambda)$, that a periodic point $p$ in $X(G(\mathcal{V}, \Sigma, \lambda))$ has a negative (positive) multiplier if there exist $I \in \mathbb{Z}$ such that $\lambda\left(p_{[I, I+\pi(p))}\right) \in \mathcal{S}^{-}\left(\mathfrak{P}, \mathcal{E}^{+} \cup \mathcal{E}^{-}\right)$ $\left(\mathcal{S}^{+}\left(\mathfrak{P}, \mathcal{E}^{+} \cup \mathcal{E}^{-}\right)\right)$. A periodic point that has neither a negative multiplier nor a positive multiplier is called neutral. For $V \in \mathcal{V}_{\mathfrak{p}}, \mathfrak{p} \in \mathfrak{P}$, denote by $P(V)$ the set of periodic points in $X(G(\mathcal{V}, \Sigma, \lambda))$ that carry the bi-infinite concatenation of a cycle $c$ at $V$ such that $\lambda(c)=\mathbf{1}_{\mathfrak{p}}$. The set of neutral periodic points is equal to $\bigcup_{\mathfrak{p} \in \mathfrak{P}} \bigcup_{V \in \mathcal{V}(\mathfrak{p})} P(V)$.

LEMMA 3.1. Let $G_{\mathcal{R}}\left(\mathfrak{P}, \mathcal{E}^{-} \cup \mathcal{E}^{+}\right)$be an $\mathcal{R}$-graph such that $\mathfrak{P} \backslash \mathfrak{P}_{\mathcal{R}}^{(1)} \neq \emptyset$ that satisfies Conditions (II) and let $G(\mathcal{V}, \Sigma, \lambda)$ be an $\mathcal{S}\left(G_{\mathcal{R}}\left(\mathfrak{P}, \mathcal{E}^{-}, \mathcal{E}^{+}\right)\right)$-presentation of a subshift $X(G(\mathcal{V}, \Sigma, \lambda))$. Then

$$
A(X(G(\mathcal{V}, \Sigma, \lambda)))=\bigcup_{\mathfrak{p} \in \mathfrak{P}} \bigcup_{V \in \mathcal{V}(\mathfrak{p})} P(V)
$$

Proof. Let

$$
p \in A(X(G(\mathcal{V}, \Sigma, \lambda)),
$$

and let $I \in \mathbb{Z}$ be such that

$$
\lambda\left(p_{[I, I+\pi(p))}\right) \in \mathcal{S}^{-}\left(\mathfrak{P}, \mathcal{E}^{-} \cup \mathcal{E}^{+}\right) \cup\left\{\mathbf{1}_{\mathfrak{p}}: \mathfrak{p} \in \mathfrak{P}\right\} \cup \mathcal{S}^{+}\left(\mathfrak{P}, \mathcal{E}^{-} \cup \mathcal{E}^{+}\right) .
$$

If

$$
\lambda\left(p_{[I, I+\pi(p))}\right) \in \mathcal{S}_{\mathcal{R}}^{-}\left(\mathfrak{P}, \mathcal{E}^{-} \cup \mathcal{E}^{+}\right),
$$

then it follows from (3.1) that $\lambda\left(p_{[I, I+\pi(p))}\right)$ is given by a cycle in the directed graph $G\left(\mathfrak{P}, \mathcal{E}_{\mathcal{R}}^{-}\right)$, contradicting Condition (II-). For the case that

$$
\lambda\left(p_{[I, I+\pi(p))}\right) \in \mathcal{S}^{+}\left(\mathfrak{P}, \mathcal{E}^{-} \cup \mathcal{E}^{+}\right),
$$

one has the symmetric argument.

For the converse, note that $\lambda\left(p_{[I, I+\pi(p))}\right)=\mathbf{1}_{\mathfrak{p}}$ implies that

$$
p \in A_{\pi(p)}(X(G(\mathcal{V}, \Sigma, \lambda))) .
$$

Given finite sets $\mathcal{E}^{-}$and $\mathcal{E}^{+}$and a relation $\mathcal{R} \subset \mathcal{E}^{-} \times \mathcal{E}^{+}$, we say that $e^{-} \in \mathcal{E}^{-}$and $\tilde{e}^{-} \in \mathcal{E}^{-}$are $\sim(\mathcal{R},-)$-equivalent if

$$
\left\{\tilde{e}^{+} \in \mathcal{E}^{+}:\left(e^{-}, \tilde{e}^{+}\right) \in \mathcal{R}\right\}=\left\{\tilde{e}^{+} \in \mathcal{E}^{+}:\left(\tilde{e}^{-}, \tilde{e}^{+}\right) \in \mathcal{R}\right\} .
$$

An equivalence relation $\sim(\mathcal{R},+)$ on $\mathcal{E}^{+}$is defined symmetrically.

Given an $\mathcal{R}$-graph $\mathcal{G}_{\mathcal{R}}\left(\mathfrak{P}, \mathcal{E}^{-} \cup \mathcal{E}^{+}\right)$, we construct an $\mathcal{R}$-graph $\mathcal{G}_{\overline{\mathcal{R}}}\left(\mathfrak{P}, \overline{\mathcal{E}}^{-} \cup \overline{\mathcal{E}}^{+}\right)$by setting

$$
\begin{aligned}
& \overline{\mathcal{E}}^{-}(\mathfrak{q}, \mathfrak{r})=\left[\mathcal{E}^{-}(\mathfrak{q}, \mathfrak{r})\right]_{\sim(\mathcal{R},-)}, \quad \overline{\mathcal{E}}^{+}(\mathfrak{q}, \mathfrak{r})=\left[\mathcal{E}^{-}(\mathfrak{q}, \mathfrak{r})\right] \sim(\mathcal{R},+), \\
& \overline{\mathcal{R}}(\mathfrak{q}, \mathfrak{r})=\left\{\left(\bar{e}^{-}, \bar{e}^{+}\right) \in \overline{\mathcal{E}}^{-}(\mathfrak{q}, \mathfrak{r}) \times \overline{\mathcal{E}}^{+}(\mathfrak{q}, \mathfrak{r}): \bar{e}^{-} \times \bar{e}^{+} \subset \mathcal{R}(\mathfrak{q}, \mathfrak{r})\right\}, \quad \mathfrak{q}, \mathfrak{r} \in \mathfrak{P} .
\end{aligned}
$$

We denote by $\overline{\mathcal{F}}_{\overline{\mathcal{R}}}^{-}$the set of edges in $\overline{\mathcal{E}}^{-}$that are the single incoming edges of their target vertices, and we denote by $\overline{\mathcal{F}}_{\overline{\mathcal{R}}}^{+}$the set of edges in $\overline{\mathcal{E}}^{+}$that are the single outgoing 
edges of their source vertices. Observe that the set $\mathfrak{P}_{\mathcal{R}}^{(1)}$ is the set of target vertices of the edges in $\overline{\mathcal{F}}_{\overline{\mathcal{R}}}^{-}$, which is equal to the set of source vertices of the edges in $\overline{\mathcal{F}}_{\overline{\mathcal{R}}}^{+}$, and that $\mathfrak{P} \backslash \mathfrak{P}_{\mathcal{R}}^{(1)}$ is the set of vertices that have at least two incoming edges in $\overline{\mathcal{E}}^{-}$or, equivalently, that have at least two outgoing edges in $\overline{\mathcal{E}}^{+}$. We set

$$
\widehat{\mathfrak{P}}=\mathfrak{P} \backslash \mathfrak{P}_{\mathcal{R}}^{(1)},
$$

and we denote by $\widehat{\mathfrak{P}}^{\bullet}$ the set of vertices in $\widehat{\mathfrak{P}}$ that are source vertices of an edge in $\overline{\mathcal{F}}_{\overline{\mathcal{R}}}^{-}$ or, equivalently, that are target vertices of an edge in $\overline{\mathcal{F}}_{\overline{\mathcal{R}}}^{+}$. For $\mathfrak{r} \in \widehat{\mathfrak{P}}^{\bullet}$, we denote by $\mathfrak{P}(\mathfrak{r})$ the set of $\mathfrak{p} \in \mathfrak{P}$ that are target vertices of a path in the graph $G\left(\mathfrak{P}, \overline{\mathcal{F}}_{\overline{\mathcal{R}}}^{-}\right)$that leaves $\mathfrak{r}$ or, equivalently, that are source vertices of a path in the graph $G\left(\mathfrak{P}, \overline{\mathcal{F}}_{\overline{\mathcal{R}}}^{+}\right)$that enters $\mathfrak{r}$. We also set

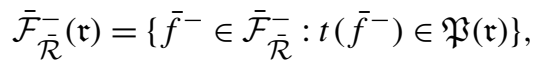

$$
\begin{aligned}
& \overline{\mathcal{F}}_{\overline{\mathcal{R}}}^{+}(\mathfrak{r})=\left\{\bar{f}^{+} \in \overline{\mathcal{F}}_{\overline{\mathcal{R}}}^{+}: s\left(\bar{f}^{+}\right) \in \mathfrak{P}(\mathfrak{r})\right\}, \quad \mathfrak{r} \in \widehat{\mathfrak{P}}^{\bullet} .
\end{aligned}
$$

For $\mathfrak{r} \in \widehat{\mathfrak{P}} \backslash \widehat{\mathfrak{P}}^{\bullet}$, we set $\mathfrak{P}(\mathfrak{r})=\emptyset$. We have obtained partitions

$$
\begin{aligned}
& \mathfrak{P}=\bigcup_{\mathfrak{r} \in \widehat{\mathfrak{P}}}(\{\mathfrak{r}\} \cup \mathfrak{P}(\mathfrak{r})), \\
& \overline{\mathcal{E}}^{-}=\left(\overline{\mathcal{E}}^{-} \backslash \overline{\mathcal{F}}_{\overline{\mathcal{R}}}^{-}\right) \cup \bigcup_{\mathfrak{r} \in \widehat{\mathfrak{P}}} \overline{\mathcal{F}}_{\overline{\mathcal{R}}}^{-}(\mathfrak{r}), \quad \overline{\mathcal{E}}^{+}=\left(\overline{\mathcal{E}}^{-} \backslash \overline{\mathcal{F}}_{\overline{\mathcal{R}}^{+}}^{+}\right) \cup \bigcup_{\mathfrak{r} \in \widehat{\mathfrak{P}}} \overline{\mathcal{F}}_{\overline{\mathcal{R}}}^{-}(\mathfrak{r}) .
\end{aligned}
$$

For all $\mathfrak{r} \in \widehat{\mathfrak{P}}^{\bullet}$, the directed graph $G\left(\{\mathfrak{r}\} \cup \mathfrak{P}(\mathfrak{r}), \overline{\mathcal{F}}_{\overline{\mathcal{R}}}^{-}(\mathfrak{r})\right)$ is an outward directed tree, the directed graph $G\left(\{\mathfrak{r}\} \cup \mathfrak{P}(\mathfrak{r}), \overline{\mathcal{F}}_{\overline{\mathcal{R}}}^{+}(\mathfrak{r})\right)$ is an inward directed tree, the directed trees $G(\{\mathfrak{r}\} \cup$ $\left.\mathfrak{P}(\mathfrak{r}), \overline{\mathcal{F}}_{\overline{\mathcal{R}}}^{-}(\mathfrak{r})\right)$ and $G\left(\{\mathfrak{r}\} \cup \mathfrak{P}(\mathfrak{r}), \overline{\mathcal{F}}_{\overline{\mathcal{R}}^{+}}^{+}(\mathfrak{r})\right)$ are reversals of one another, and the set $\mathfrak{L}(\mathfrak{r})$ of their leaves is given by the set of vertices in $\mathfrak{P}(\mathfrak{r})$ that are source vertices of an edge in $\overline{\mathcal{E}}^{-} \backslash$ $\overline{\mathcal{F}}^{-}$or, equivalently, that are target vertices of an edge in $\overline{\mathcal{E}}^{+} \backslash \overline{\mathcal{F}}^{+}$. To a vertex $\mathfrak{r} \in \mathfrak{P} \backslash \mathfrak{P}^{\bullet}$ we associate the degenerate tree $G(\{\mathfrak{r}\}, \emptyset)$ with its leaf $\mathfrak{r}$. For $\mathfrak{r} \in \widehat{\mathfrak{P}}$, if the source (target) vertex of an edge $e^{-} \in \overline{\mathcal{E}}^{-} \backslash \overline{\mathcal{F}}_{\overline{\mathcal{R}}}^{-}\left(e^{+} \in \overline{\mathcal{E}}^{+} \backslash \overline{\mathcal{F}}_{\overline{\mathcal{R}}}^{-}\right)$is in $\mathfrak{P}(\mathfrak{r})$, then this source (target) vertex is necessarily in $\mathfrak{L}(\mathfrak{r})$.

From the $\mathcal{R}$-graph $\mathcal{G}_{\overline{\mathcal{R}}}\left(\mathfrak{P}, \overline{\mathcal{E}}^{-} \cup \overline{\mathcal{E}}^{+}\right)$, we construct an $\mathcal{R}$-graph $\mathcal{G}_{\widehat{\mathcal{R}}}\left(\widehat{\mathfrak{P}}, \widehat{\mathcal{E}}^{-} \cup \widehat{\mathcal{E}}^{+}\right)$. We postulate that there are bijections

$$
\bar{e}^{-} \rightarrow \widehat{\bar{e}^{-}} \in \widehat{\mathcal{E}}^{-}\left(\bar{e}^{-} \in \overline{\mathcal{E}}^{-} \backslash \overline{\mathcal{F}}^{-}\right), \quad \bar{e}^{+} \rightarrow \widehat{\bar{e}^{+}} \in \widehat{\mathcal{E}}^{+}\left(\bar{e}^{+} \in \overline{\mathcal{E}}^{+} \backslash \overline{\mathcal{F}}^{+}\right) .
$$

We specify the source and target mappings of the graph $\mathcal{G}_{\widehat{\mathcal{R}}}\left(\widehat{\mathfrak{P}}, \widehat{\mathcal{E}}^{-} \cup \hat{\mathcal{E}}^{+}\right)$by setting

$$
\begin{array}{ll}
t\left(\widehat{\bar{e}^{-}}\right)=t\left(\bar{e}^{-}\right), & \bar{e}^{-} \in \overline{\mathcal{E}}^{-} \backslash \overline{\mathcal{F}}^{-}, \\
s\left(\widehat{\bar{e}^{+}}\right)=s\left(\bar{e}^{+}\right), & \bar{e}^{+} \in \overline{\mathcal{E}}^{+} \backslash \overline{\mathcal{F}}^{+},
\end{array}
$$

and by assigning for $\mathfrak{r} \in \widehat{\mathfrak{P}}$ and for $\bar{e}^{-} \in \overline{\mathcal{E}}^{-}\left(\bar{e}^{+} \in \overline{\mathcal{E}}^{+}\right)$to the edge $\widehat{\bar{e}^{-}}\left(\widehat{\overline{e^{+}}}\right)$the vertex $\mathfrak{r}$ as source (target) vertex. We set

$$
\left.\left.\widehat{\mathcal{R}}=\left\{\left(\widehat{\bar{e}^{-}}, \widehat{\bar{e}^{+}}\right) \in \widehat{\mathcal{E}}^{-} \times \widehat{\mathcal{E}}^{+}:\left(\bar{e}^{-}, \bar{e}^{+}\right) \in \overline{\mathcal{R}} \cap\left(\left(\overline{\mathcal{E}}^{-} \backslash \overline{\mathcal{F}}^{-}\right) \times \overline{\mathcal{E}}^{+} \backslash \overline{\mathcal{F}}^{+}\right)\right)\right)\right\} .
$$

In the $\mathcal{R}$-graph $\mathcal{G}_{\widehat{\mathcal{R}}}\left(\widehat{\mathfrak{P}}, \widehat{\mathcal{E}}^{-} \cup \widehat{\mathcal{E}}^{+}\right)$, every vertex has at least two incoming edges in $\widehat{\mathcal{E}}^{-}$, which means that every vertex in $\widehat{\mathcal{E}}^{+}$has at least two outgoing edges in $\widehat{\mathcal{E}}^{+}$. 
We introduce additional notation for subshifts. We denote the set of periodic points in $A(X)$ by $P(A(X))$. The subshifts $X \subset \Sigma^{\mathbb{Z}}$ that we consider in this paper are such that $P(A(X))$ is dense in $X$. We introduce a pre-order relation $\gtrsim$ into the set $P(A(X))$. For $q, r \in P(A(X)), q \gtrsim r$ means that there exists a point in $A(X)$ that is left asymptotic to the orbit of $q$ and right asymptotic to the orbit of $r$. The equivalence relation on $P(A(X))$ that results from the pre-order relation $\gtrsim$ we denote by $\approx$.

LEMMA 3.2. Let $G_{\mathcal{R}}\left(\mathfrak{P}, \mathcal{E}^{-} \cup \mathcal{E}^{+}\right)$be an $\mathcal{R}$-graph such that $\mathfrak{P} \backslash \mathfrak{P}_{\mathcal{R}}^{(1)} \neq \emptyset$ that satisfies Conditions (II) and let $G(\mathcal{V}, \Sigma, \lambda)$ be an $\mathcal{S}\left(G_{\mathcal{R}}\left(\mathfrak{P}, \mathcal{E}^{-}, \mathcal{E}^{+}\right)\right)$-presentation of a subshift $X(G(\mathcal{V}, \Sigma, \lambda))$. For $\mathfrak{q}, \mathfrak{q}^{\prime} \in \mathfrak{P}, \mathfrak{q} \neq \mathfrak{q}^{\prime}$,

$$
\left(\bigcup_{V \in \mathcal{V}(\mathfrak{q})} P(V)\right) \cap\left(\bigcup_{V \in \mathcal{V}\left(\mathfrak{q}^{\prime}\right)} P(V)\right)=\emptyset .
$$

Proof. Let $\mathfrak{p}, \mathfrak{p}^{\prime} \in \mathfrak{P}, V \in \mathcal{V}(\mathfrak{p}), V^{\prime} \in \mathcal{V}\left(\mathfrak{p}^{\prime}\right), q \in P(V), q^{\prime} \in P\left(V^{\prime}\right)$ and assume that $q=q^{\prime}$. We show that

$$
\mathfrak{p}=\mathfrak{p}^{\prime}
$$

Let $c\left(c^{\prime}\right)$ be simple cycles such that

$$
\lambda(c)=\mathbf{1}_{\mathfrak{p}}, \quad \lambda\left(c^{\prime}\right)=\mathbf{1}_{\mathfrak{p} \prime},
$$

whose bi-infinite concatenations are carried by $q$. Let $\pi$ denote the period of $q$. Let $I \in \mathbb{Z}$ be such that

$$
q_{[I, I+\pi)}=c,
$$

and let $I^{\prime} \in[I, I+\pi)$ be such that

$$
q_{\left[I^{\prime}, I^{\prime}+\pi\right)}=c^{\prime} .
$$

In the case that $I^{\prime}=I,(3.3)$ implies (3.2) and in the case that $I^{\prime}>I$, (3.3) together with (3.4) and (3.5) imply that

$$
\left\{\lambda\left(c_{\left[I, I^{\prime}\right)}, c_{\left[I^{\prime}, I+\pi\right)}^{\prime}\right\} \in\left\{\mathbf{1}_{\mathfrak{p}}: \mathfrak{p} \in \mathfrak{P}\right\} \cup \mathcal{S}^{+}\left(G_{\mathcal{R}}\left(\mathfrak{P}, \mathcal{E}^{-}, \mathcal{E}^{+}\right)\right),\right.
$$

and, more precisely, that

$$
\lambda\left(c_{\left[I, I^{\prime}\right)}\right)=\mathbf{1}_{\mathfrak{p}}, \quad \lambda\left(c_{\left[I^{\prime}, I+\pi\right)}^{\prime}\right)=\mathbf{1}_{\mathfrak{p}^{\prime}},
$$

which gives

$$
\mathbf{1}_{\mathfrak{p}} \mathbf{1}_{\mathfrak{p}^{\prime}}=\mathbf{1}_{\mathfrak{p}},
$$

and (3.3) is shown.

LEMMA 3.3. Let $G_{\mathcal{R}}\left(\mathfrak{P}, \mathcal{E}^{-} \cup \mathcal{E}^{+}\right)$be an $\mathcal{R}$-graph such that $\mathfrak{P} \backslash \mathfrak{P}_{\mathcal{R}}^{(1)} \neq \emptyset$ that satisfies Conditions (II) and let $G(\mathcal{V}, \Sigma, \lambda)$ be an $\mathcal{S}\left(G_{\mathcal{R}}\left(\mathfrak{P}, \mathcal{E}^{-}, \mathcal{E}^{+}\right)\right)$-presentation of a subshift $X(G(\mathcal{V}, \Sigma, \lambda))$. Let

$$
\mathfrak{r}(-), \mathfrak{r}(+) \in \widehat{\mathfrak{P}} \text {. }
$$

Let

$$
V(-) \in \mathcal{V}(\mathfrak{r}(-)), \quad V(+) \in \mathcal{V}(\mathfrak{r}(+))
$$

and let

$$
p(-) \in P(V(-)), \quad p(+) \in P(V(+)) .
$$

If $p(-) \gtrsim p(+)$ or if $p(+) \lesssim p(-)$, then $\mathfrak{r}(-)=\mathfrak{r}(+)$. 
Proof. We derive a contradiction from the assumptions that $p(-) \gtrsim p(+)$ and that

$$
\mathfrak{r}(-) \neq \mathfrak{r}(+) .
$$

Let $c(-)(c(+))$ be the simple cycle whose bi-infinite concatenation is carried by $p(-)$ $(p(+))$. Let $x \in A(X(G(\mathcal{V}, \Sigma, \lambda)))$ be left asymptotic to $p(-)$ and right asymptotic to $p(+)$. By Lemma 3.2,

$$
p(-) \neq p(+) .
$$

Let $I(-)(I(+))$ denote the maximal (minimal) index such that $x_{(-\infty, I(-))}\left(x_{[I(+), \infty)}\right)$ carries the left (right) infinite concatenation of $c(-)(c(+))$. From (3.6), it is seen that $I(-)<I(+)$. Write

$$
\begin{aligned}
& u^{+}\left(\lambda\left(x_{(I(-), I(+))}\right)=\left(\prod_{0<k(+) \leq K(+)} e_{k(+)}^{+}\right) \mathbf{1}_{\mathfrak{q}\left(\lambda\left(x_{(I(-), I(+))}\right)\right.},\right. \\
& u^{-\left(\lambda\left(x_{(I(-), I(+))}\right)\right.}=\mathbf{1}_{\mathfrak{q}\left(\lambda\left(x_{(I(-), I(+))}\right)\right.}\left(\prod_{0<k(-) \leq K(-)} e_{k(-)}^{-}\right) .
\end{aligned}
$$

Assume that $K(+)>0$. The source vertex of $e^{+}(K(+))$ is $\mathfrak{r}(-)$, which has more than one outgoing edge in $G\left(\mathfrak{P}, \mathcal{E}^{+}\right)$, which implies that $x \notin A^{-}(X(G(\mathcal{V}, \Sigma, \lambda)))$. If $K(+)=0$, then one has by (3.6) that $K(-)>0$. The target vertex of $e^{-}(K(-))$ is $\mathfrak{r}(+)$, which has more than one incoming edge in $G\left(\mathfrak{P}, \mathcal{E}^{-}\right)$, which implies that $x \notin$ $A^{+}(X(G(\mathcal{V}, \Sigma, \lambda)))$.

The proof under the assumption that $p(-) \lesssim p(-)$ is symmetric.

The following theorem is proved for Markov-Dyck shifts in [KM2].

THEOREM 3.4. Let $\mathcal{G}_{\mathcal{R}}\left(\mathfrak{P}, \mathcal{E}^{-}, \mathcal{E}^{+}\right)$be an $\mathcal{R}$-graph such that $\mathfrak{P} \backslash \mathfrak{P}_{\mathcal{R}}^{(1)} \neq \emptyset$ that satisfies Conditions (I), (II) and (III), and let $G(\mathcal{V}, \Sigma, \lambda)$ be an $\mathcal{S}\left(G_{\mathcal{R}}\left(\mathfrak{P}, \mathcal{E}^{-}, \mathcal{E}^{+}\right)\right)$-presentation of a subshift $X(G(\mathcal{V}, \Sigma, \lambda))$. Let $p \in \mathcal{V}(\mathfrak{p}), \mathfrak{p} \in \mathfrak{P}$, and $p^{\prime} \in \mathcal{V}\left(\mathfrak{p}^{\prime}\right), \mathfrak{p}^{\prime} \in \mathfrak{P}$. For $p$ and $p^{\prime}$ to be $\approx$-equivalent, it is necessary and sufficient that $\mathfrak{p}$ and $\mathfrak{p}^{\prime}$ are in the same element of the partition $\{\{\mathfrak{r}\} \cup \mathfrak{P}(\mathfrak{r}), \mathfrak{r} \in \widehat{\mathfrak{P}}\}$.

Proof. Choose for $\mathfrak{r} \in \widehat{\mathfrak{P}}$ a vertex $V_{\mathfrak{r}} \in \mathcal{V}(\mathfrak{r})$ and a cycle $c_{\mathfrak{r}}$ at $V_{\mathfrak{r}}$ such that $\lambda\left(c_{\mathfrak{r}}\right)=\mathbf{1}_{\mathfrak{r}}$. Then choose a periodic point $r$ that carries the bi-infinite concatenation of $c_{\mathfrak{r}}$. Let $c(\mathfrak{p})$ be the simple cycle at $V(\mathfrak{p})$ that has its bi-infinite concatenation carried by $p$. Choose a path $\left(e_{i(-)}^{-}\right)_{1 \leq i(-) \leq I(-)}$ in $G\left(\mathfrak{P}, \mathcal{E}_{\mathcal{R}}^{-}\right)$, with source vertex $s\left(e_{1}^{-}\right)$equal to $\mathfrak{r}$ and with target vertex $t\left(e_{I(-)}^{-}\right)$equal to $\mathfrak{p}$, and choose a path $f(-)$ in $G(\mathcal{V}, \Sigma, \lambda)$ from $V_{\mathfrak{r}}$ to $V(\mathfrak{p})$ such that $\lambda(f(-))=\prod_{1 \leq i(-) \leq I(-)} e_{i(-)}^{-}$. Choose also a path $\left(e_{i(+)}^{+}\right)_{1 \leq i(+) \leq I(+)}$ in $G\left(\mathfrak{P}, \mathcal{E}_{\mathcal{R}}^{+}\right)$, with source vertex $s\left(e_{1}^{+}\right)$equal to $\mathfrak{p}$ and with target vertex $t\left(e_{I(+)}^{-}\right)$equal to $\mathfrak{r}$, and choose a path $f(+)$ in $G(\mathcal{V}, \Sigma, \lambda)$ from $V(\mathfrak{p})$ to $V_{\mathfrak{r}}$ such that $\lambda(f(+))=\prod_{1 \leq i(+) \leq I(+)} e_{i(+)}^{+}$. The periodic points $p$ and $r$ are $\approx$-equivalent: points in $A(X(G(\mathcal{V}, \Sigma, \lambda)))$ that are left asymptotic to $p$ and right asymptotic to $r$ are given by the points that carry a left infinite concatenation of $c(\mathfrak{p})$, followed by the path $f(+)$, followed by the right infinite concatenation of $c_{\mathfrak{r}}$, and points in $A(X(G(\mathcal{V}, \Sigma, \lambda)))$ that are left asymptotic to $r$ and right asymptotic to $p$ are given by the points that carry a left infinite concatenation of $c_{\mathfrak{r}}$, 
followed by the path $f(-)$, followed by the right infinite concatenation of $c(\mathfrak{p})$. This proves sufficiency.

To prove necessity, apply Lemma 3.3.

We recall at this point from [Kr1] the construction of the semigroup $\mathcal{S}(X)$ for a subshift $X$ with Property $(A)$. Given a subshift $X \subset \Sigma^{\mathbb{Z}}$ with Property $(A)$, we denote by $Y(X)$ the set of points in $X$ that are left asymptotic to a point in $P(A(X))$ and also right asymptotic to a point in $P(A(X))$. Let $y, \tilde{y} \in Y(X)$, let $y$ be left asymptotic to $q \in P(A(X))$ and right asymptotic to $r \in P(A(X))$ and let $\tilde{y}$ be left asymptotic to $\tilde{q} \in P(A(X))$ and right asymptotic to $\tilde{r} \in P(A(X))$. Given that $X$ has the properties $\left(a, n, H_{n}\right), n \in \mathbb{N}$, we say that $y$ and $\tilde{y}$ are equivalent, $y \approx \tilde{y}$, if $q \approx \tilde{q}$ and $r \approx \tilde{r}$ and, if for $n \in \mathbb{N}$ such that $q, r, \tilde{q}, \tilde{r} \in$ $P\left(A_{n}(X)\right)$ and for $I, J, \tilde{I}, \tilde{J} \in \mathbb{Z}, I<J, \tilde{I}<\tilde{J}$ such that

$$
\begin{array}{ll}
y_{(-\infty, I]}=q_{(-\infty, 0]}, & y_{(J, \infty)}=r_{(0, \infty)}, \\
\tilde{y}_{(-\infty, \tilde{I}]}=\tilde{q}_{(-\infty, 0]}, & \tilde{y}_{(\tilde{J}, \infty)}=\tilde{r}_{(0, \infty)},
\end{array}
$$

one has for $h \geq 3 H_{n}$ and for

$$
a \in X_{(I-h, J+h]}, \quad \tilde{a} \in X_{(\tilde{I}-h, \tilde{J}+h]},
$$

such that

$$
\begin{aligned}
a_{\left(I-H_{n}, J+H_{n}\right]}= & y_{\left(I-H_{n}, J+H_{n}\right]}, \quad \tilde{a}_{\left(\tilde{I}-H_{n}, \tilde{J}+H_{n}\right]}=\tilde{y}_{\left(\tilde{I}-H_{n}, \tilde{J}+H_{n}\right]}, \\
& a_{\left(I-h, I-h+H_{n}\right)}=\tilde{a}_{\left(\tilde{I}-h, \tilde{I}-h+H_{n}\right)}, \\
& a_{\left(J+h-H_{n}, J+h\right]}=\tilde{a}_{\left(\tilde{J}+h-H_{n}, \tilde{J}+h\right]},
\end{aligned}
$$

and such that

$$
\begin{array}{ll}
a_{(I-h, I]} \in A_{n}(X)_{(I-h, I]}, & \tilde{a}_{(\tilde{J}-h, \tilde{I}]} \in A_{n}(X)_{(\tilde{J}-h, \tilde{I}]}, \\
a_{(J, J+h]} \in A_{n}(X)_{(J, J+h]}, & \tilde{a}_{(\tilde{J}, \tilde{J}+h]} \in A_{n}(X)_{(\tilde{J}, \tilde{J}+h]},
\end{array}
$$

that $a$ and $\tilde{a}$ have the same context. To give $[Y(X)] \approx$ the structure of a semigroup, let $u, v \in Y(X)$, let $u$ be right asymptotic to $q \in P(A(X))$ and let $v$ be left asymptotic to $r \in P(A(X))$. If here $q \gtrsim r$, then $[u]_{\approx}[v]_{\approx}$ is set equal to $[y] \approx$, where $y$ is any point in $Y$ such that there are $n \in \mathbb{N}, I, J, \hat{I}, \hat{J} \in \mathbb{Z}, I<J, \hat{I}<\hat{J}$, such that $q, r \in A_{n}(X)$, and such that

$$
\begin{gathered}
u_{(I, \infty)}=q_{(I, \infty)}, \quad v_{(-\infty, J]}=r_{(-\infty, J]}, \\
y_{\left(-\infty, \hat{I}+H_{n}\right]}=u_{\left(-\infty, I+H_{n}\right]}, \quad y_{\left(\hat{J}-H_{n}, \infty\right)}=v_{\left(J-H_{n}, \infty\right)},
\end{gathered}
$$

and

$$
y_{(\hat{I}, \hat{J}]} \in A_{n}(X)_{(\hat{I}, \hat{J}]},
$$

provided that such a point $y$ exists. If such a point $y$ does not exist, $[u]_{\approx}[v] \approx$ is set equal to zero. Also, in the case that one does not have $q \gtrsim r,[u] \approx[v] \approx$ is set equal to zero.

THEOREM 3.5. Let $G_{\mathcal{R}}\left(\mathfrak{P}, \mathcal{E}^{-} \cup \mathcal{E}^{+}\right)$be an $\mathcal{R}$-graph such that

$$
\mathfrak{P} \backslash \mathfrak{P}_{\mathcal{R}}^{(1)} \neq \emptyset,
$$


that satisfies Conditions (I), (II) and (III), and let a subshift $X(G(\mathcal{V}, \Sigma, \lambda))$ be given by an $\mathcal{S}\left(G_{\mathcal{R}}\left(\mathfrak{P}, \mathcal{E}^{+} \cup \mathcal{E}^{-}\right)\right)$-presentation $G(\mathcal{V}, \Sigma, \lambda)$. Then

$$
\mathcal{S}(X(G(\mathcal{V}, \Sigma, \lambda)))=\mathcal{S}\left(G_{\widehat{\mathcal{R}}}\left(\widehat{\mathfrak{P}}, \widehat{\mathcal{E}}^{-} \cup \widehat{\mathcal{E}}^{+}\right)\right),
$$

and $X\left(G_{\mathcal{R}}\left(\mathfrak{P}, \mathcal{E}^{+} \cup \mathcal{E}^{-}\right)\right)$is given by an $\mathcal{S}\left(G_{\widehat{\mathcal{R}}}\left(\widehat{\mathfrak{P}}, \widehat{\mathcal{E}}^{+} \cup \widehat{\mathcal{E}}^{-}\right)\right)$-presentation.

Proof. We choose for $\mathfrak{r} \in \widehat{\Re}$ a periodic point $y^{\circ}(\mathfrak{r}) \in X(\mathfrak{P}, \Sigma, \lambda)$ such that

$$
\lambda\left(\left[0, \pi\left(y^{\circ}(\mathfrak{r})\right)\right)=\mathbf{1}_{\mathfrak{r}} .\right.
$$

By Lemma 3.1 and by Theorem 3.2, we can choose a system of representatives $Y^{\circ}$ of the equivalence relation $\approx$ such that $Y^{\circ} \supset\left\{y^{\circ}(\mathfrak{r}): \mathfrak{r} \in \mathfrak{R}_{\widehat{\mathcal{R}}}\right\}$, and such that every point in $Y^{\circ}$ is left asymptotic to a point in $\left\{y^{\circ}(\mathfrak{r}): \mathfrak{r} \in \mathfrak{R}_{\widehat{\mathcal{R}}}\right\}$ and also right asymptotic to a point in $\left\{y^{\circ}(\mathfrak{r}): \mathfrak{r} \in \mathfrak{R}_{\widehat{\mathcal{R}}}\right\}$.

We set

$$
\begin{aligned}
& \varphi\left(e^{-}\right)=\left[e^{-} \widehat{] \sim(\mathcal{R},-)}, \quad e^{-} \in \mathcal{E}^{-},\right. \\
& \varphi\left(f^{-}\right)=\mathbf{1}_{\mathfrak{r}}, \quad\left[f^{-}\right]_{\sim(\mathcal{R},-)} \in \overline{\mathcal{F}}_{\overline{\mathcal{R}}}^{-}(\mathfrak{r}), \mathfrak{r} \in \widehat{\mathfrak{R}}, \\
& \varphi\left(\mathbf{1}_{\mathfrak{p}}\right)=\mathbf{1}_{\mathfrak{r}}, \quad \mathfrak{p} \in \mathfrak{P}_{\mathfrak{r}}, \mathfrak{r} \in \widehat{\mathfrak{R}}, \\
& \varphi\left(f^{+}\right)=\mathbf{1}_{\mathfrak{r}}, \quad\left[f^{+}\right]_{\sim(\mathcal{R},+)} \in \overline{\mathcal{F}}_{\overline{\mathcal{R}}}^{+}(\mathfrak{r}), \mathfrak{r} \in \widehat{\mathfrak{R}}, \\
& \varphi\left(e^{+}\right)=\left[e^{+}\right]_{\sim(\mathcal{R},+)} \quad e^{+} \in \mathcal{E}^{+},
\end{aligned}
$$

and, for

$$
g(-) \in \mathcal{S}^{-}\left(G_{\mathcal{R}}\left(\mathfrak{P}, \mathcal{E}^{-} \cup \mathcal{E}^{+}\right)\right), \quad g(+) \in \mathcal{S}^{+}\left(G_{\mathcal{R}}\left(\mathfrak{P}, \mathcal{E}^{-} \cup \mathcal{E}^{+}\right)\right),
$$

we set

$$
\varphi\left(g^{-}\right)=\prod_{1 \leq i(-) \leq \ell(g(-))} \varphi\left(e_{i(-)}[g(-)]\right), \quad \varphi\left(g^{+}\right)=\prod_{1 \leq i(+) \leq \ell(g(+))} \varphi\left(e_{i(+)}[g(+)]\right) .
$$

We set

$$
\widehat{\lambda}(\sigma)=\varphi(\lambda(\sigma)), \quad \sigma \in \Sigma,
$$

and

$$
\begin{aligned}
& J_{-}\left(y^{\circ}\right)=\max \left\{J<0: y_{(-\infty, J]}^{\circ} \in\left\{y^{\circ}(\mathfrak{r})_{(-\infty, J]}: \mathfrak{r} \in \widehat{\Re}\right\}\right\}, \\
& J_{-}\left(y^{\circ}\right)=\min \left\{J>0: y_{[J,-\infty)}^{\circ} \in\left\{y^{\circ}(\mathfrak{r})_{[J,-\infty)}: \mathfrak{r} \in \widehat{\mathfrak{R}}\right\}, \quad y^{\circ} \in Y^{\circ} .\right.
\end{aligned}
$$

An isomorphism $\Psi$ of $\mathcal{S}\left(X(\mathcal{V}, \Sigma, \lambda)\right.$ onto $\left(\mathcal{S}\left(G_{\widehat{\mathcal{R}}}\left(\widehat{\mathfrak{P}}, \widehat{\mathcal{E}}^{+} \cup \widehat{\mathcal{E}}^{-}\right)\right)\right)$is given by

$$
\Psi\left(\left[\left(y^{\circ}\right)\right] \approx\right)=\prod_{J_{-}\left(y^{\circ}\right)<j<J_{+}\left(y^{\circ}\right)} \widehat{\lambda}\left(y_{j}^{\circ}\right), \quad y^{\circ} \in Y^{\circ} .
$$




\section{Examples}

Consider an $\mathcal{R}$-graph $G_{\mathcal{R}}\left(\mathfrak{P}, \mathcal{E}^{-} \cup \mathcal{E}^{+}\right)$such that

$$
\mathfrak{P} \backslash \mathfrak{P}_{\mathcal{R}}^{(1)} \neq \emptyset
$$

that satisfies Conditions (I), (II) and (III). We say that a periodic point

$$
p \in X\left(G_{\mathcal{R}}\left(\mathfrak{P}, \mathcal{E}^{-} \cup \mathcal{E}^{+}\right)\right)
$$

has multiplier $\widehat{e}^{-} \in \widehat{\mathcal{E}}^{-}\left(\widehat{e}^{+} \in \widehat{\mathcal{E}}^{+}\right)$if there is an $I \in \mathbb{Z}$ such that $\prod_{I \leq i<I+\pi(p)} \widehat{p}_{i}$ is equal to a power of $\widehat{e}^{-}\left(\widehat{e}^{+}\right)$. For $\widehat{e}^{-} \in \widehat{\mathcal{E}}^{-}\left(\widehat{e}^{+} \in \widehat{\mathcal{E}}^{+}\right)$, we denote by $\Lambda\left(\widehat{e}^{-}\right)\left(\Lambda\left(\widehat{e}^{+}\right)\right)$the minimal period $\pi$ such that $X\left(G_{\mathcal{R}}\left(\mathfrak{P}, \mathcal{E}^{-} \cup \mathcal{E}^{+}\right)\right)$has a periodic point of period $\pi$ with multiplier $\widehat{e}^{-}\left(\widehat{e}^{+}\right)$. The mappings $\widehat{e}^{-} \rightarrow \Lambda\left(\widehat{e}^{-}\right)\left(\widehat{e}^{-} \in \widehat{\mathcal{E}}^{-}\right)$and $\widehat{e}^{+} \rightarrow \Lambda\left(\widehat{e}^{+}\right)\left(\widehat{e}^{+} \in\right.$ $\left.\widehat{\mathcal{E}}^{+}\right)$are invariantly associated to $X\left(G_{\mathcal{R}}\left(\mathfrak{P}, \mathcal{E}^{-} \cup \mathcal{E}^{+}\right)\right)$[HIK, §4].

4.1. One-vertex $\mathcal{R}$-graphs. In this subsection, we consider one-vertex $\mathcal{R}$-graphs

$$
G=G_{\mathcal{R}}\left(\{\mathfrak{p}\}, \mathcal{E}^{-} \cup \mathcal{E}^{+}\right)
$$

that satisfy Conditions (I), (II) and (III) such that $X(G)$ is not of finite type. Given the graph $G$, we denote the set of fixed points of $X(G)$ with negative (positive) multiplier by $P_{1}^{-}(X(G))\left(P_{1}^{+}(X(G))\right)$ and we define a relation

$$
\mathcal{Q}_{1}(X(G)) \subset P_{1}^{-}(X(G)) \times P_{1}^{+}(X(G))
$$

as the relation that contains the pairs

$$
(p(-), p(+)) \in P_{1}^{-}(X(G)) \times P_{1}^{-}(X(G))
$$

such that $X(G)$ has a point that is negatively asymptotic to $p(-)$ and positively asymptotic to $p(+)$.

THEOREM 4.1. Let

$$
G=G_{\mathcal{R}}\left(\{\mathfrak{p}\}, \mathcal{E}^{-} \cup \mathcal{E}^{+}\right)
$$

be an $\mathcal{R}$-graph that satisfies Conditions (I), (II) and (III) such that $X(G)$ is not of finite type and let

$$
\widetilde{G}=G_{\widetilde{\mathcal{R}}}\left(\widetilde{\mathfrak{P}}, \widetilde{\mathcal{E}}^{-} \cup \widetilde{\mathcal{E}}^{+}\right)
$$

be an $\mathcal{R}$-graph such that the subshift $X(\widetilde{G})$ is topologically conjugate to the subshift $X(G)$. Then the $\mathcal{R}$-graphs $\widetilde{G}$ and $G$ are isomorphic.

Proof. The hypothesis that the subshifts $X(\widetilde{G})$ and $X(G)$ are topologically conjugate implies that

$$
\begin{array}{ll}
\Lambda\left(\widetilde{e}^{-}\right)=1, & \widetilde{e}^{-} \in \widetilde{\mathcal{E}}^{-}, \\
\Lambda\left(\widetilde{e}^{+}\right)=1, & \widetilde{e}^{+} \in \widetilde{\mathcal{E}}^{+} .
\end{array}
$$

This means that $\widetilde{G}$ is a one-vertex $\mathcal{R}$-graph. This hypothesis also implies that $\widetilde{G}$ satisfies Conditions (I), (II) and (III), and that the relations $\mathcal{Q}_{1}(X(\widetilde{G}))$ and $\mathcal{Q}_{1}(X(G))$ are isomorphic. The relations $\widetilde{\mathcal{R}}$ and $\mathcal{Q}_{1}(X(\widetilde{G}))$ are isomorphic and so are the relations $\widetilde{\mathcal{R}}$ and $\mathcal{Q}_{1}(X(\widetilde{G}))$. 
4.2. A family of two-vertex $\mathcal{R}$-graphs. In this subsection, we consider two-vertex $\mathcal{R}$-graphs

$$
G=G_{\mathcal{R}}\left(\{\mathfrak{P}\}, \mathcal{E}^{-} \cup \mathcal{E}^{+}\right), \quad \mathfrak{P}=\{\mathfrak{p}, \mathfrak{q}\},
$$

that satisfy Conditions (I), (II) and (III) such that

$$
\mathfrak{P}_{\mathcal{R}}^{(1)}=\{\mathfrak{q}\} .
$$

Given the $\mathcal{R}$-graph $G$, we set

$$
\widehat{G}=G_{\widehat{\mathcal{R}}}\left(\{\widehat{\mathfrak{p}}\}, \widehat{\mathcal{E}}^{-} \cup \widehat{\mathcal{E}}^{+}\right),
$$

and we set

$$
\widehat{\mathcal{E}}_{1}^{-}=\left\{\widehat{e}^{-} \in \widehat{\mathcal{E}}^{-}: \Lambda\left(\widehat{e}^{-}\right)=1\right\}, \quad \widehat{\mathcal{E}}_{1}^{+}=\left\{\widehat{e}^{+} \in \widehat{\mathcal{E}}^{+}: \Lambda\left(\widehat{e}^{+}\right)=1\right\} .
$$

One notes that

$$
\widehat{\mathcal{E}}_{1}^{-}=\left\{\widehat{e^{-}}: e^{-} \in \mathcal{E}^{-}(\mathfrak{p}, \mathfrak{p})\right\}, \quad \widehat{\mathcal{E}}_{1}^{+}=\left\{\widehat{e^{+}}: e^{+} \in \mathcal{E}^{+}(\mathfrak{p}, \mathfrak{p})\right\} .
$$

We denote by $P_{1}^{-}(X(G))\left(P_{1}^{+}(X(G))\right)$ the set of fixed points of $X$ that have a multiplier in $\widehat{\mathcal{E}}_{1}^{-}\left(\widehat{\mathcal{E}}_{1}^{+}\right)$and we define a relation

$$
\mathcal{Q}_{1}(X(G)) \subset P_{1}^{-}(X(G)) \times P_{1}^{+}(X(G))
$$

as the set of pairs

$$
(p(-), p(+)) \in P_{1}^{-}(X(G)) \times P_{1}^{+}(X(G))
$$

such that $X$ has a point that is left asymptotic to $p(-)$ and right asymptotic to $p(+)$. This relation $\mathcal{Q}_{1}(X(G))$ is an invariant of topological conjugacy. We set

$$
\widehat{\mathcal{E}}_{2}^{-}(X(G))=\left\{\widehat{e}^{-} \in \widehat{\mathcal{E}}^{-}: \Lambda\left(\widehat{e}^{-}\right)=2\right\}, \quad \widehat{\mathcal{E}}_{2}^{+}(X(G))=\left\{\widehat{e}^{+} \in \widehat{\mathcal{E}}^{+}: \Lambda\left(\widehat{e}^{+}\right)=2\right\} .
$$

One notes that

$$
\widehat{\mathcal{E}}_{2}^{-}(X(G))=\left\{\widehat{e^{-}}: e^{-} \in \mathcal{E}^{-}(\mathfrak{q}, \mathfrak{p})\right\}, \quad \widehat{\mathcal{E}}_{2}^{+}(X(G))=\left\{\widehat{e^{+}}: e^{+} \in \mathcal{E}^{+}(\mathfrak{q}, \mathfrak{p})\right\} .
$$

For $\widehat{e} \in \widehat{\mathcal{E}}_{2}^{-}\left(\widehat{e}^{-} \in \widehat{\mathcal{E}}_{2}^{+}\right)$, we denote by $\mathcal{O}_{2}^{\left(\widehat{e}^{-}\right)}\left(\mathcal{O}_{2}^{\left(\widehat{e}^{+}\right)}\right)$the set of orbits of length two in $X(G)$ with multiplier $\widehat{e}^{-}\left(\widehat{e}^{+}\right)$. We set

$$
\begin{aligned}
& \rho^{-}(X(G))=\operatorname{gcd}\left\{\operatorname{card}\left(\mathcal{O}_{2}^{(\widehat{e})}\right): \widehat{e} \in \widehat{\mathcal{E}}_{2}^{-}\right\}, \\
& \rho^{+}(X(G))=\operatorname{gcd}\left\{\operatorname{card}\left(\mathcal{O}_{2}^{\left(\widehat{e}^{+}\right)}\right): \widehat{e}^{+} \in \widehat{\mathcal{E}}_{2}^{+}\right\},
\end{aligned}
$$

and

$$
\mathcal{O}_{2}^{-}(X(G))=\bigcup_{\widehat{e}^{-} \in \mathcal{O}_{2}^{\left(\widehat{e}^{-}\right)}} \mathcal{O}_{2}^{\left(\widehat{e}^{-}\right)}, \quad \mathcal{O}_{2}^{+}(X(G))=\bigcup_{\widehat{e}^{+} \in \mathcal{O}_{2}^{\left(\widehat{e}^{+}\right)}} \mathcal{O}_{2}^{\left(\widehat{e}^{+}\right)} .
$$

We define a relation

$$
\mathcal{Q}_{2}(X(G)) \subset \mathcal{O}_{2}^{-}(X(G)) \times \mathcal{O}_{2}^{+}(X(G))
$$

as the relation that contains the pairs

$$
(o(-), o(+)) \in \mathcal{O}_{2}^{-}(X(G)) \times \mathcal{O}_{2}^{+}(X(G))
$$


such that $X$ has an orbit that is left asymptotic to $o(-)$ and right asymptotic to $o(+)$. This relation $\mathcal{Q}_{2}(X(G))$ is an invariant of topological conjugacy.

We set

$$
\begin{aligned}
& \alpha(X(G))=\frac{1}{2}\left(I_{2}^{(0)}(X(G))-\operatorname{card}\left(\mathcal{Q}_{1}(X(G))\right),\right. \\
& \beta(X(G))=\operatorname{card}\left(\mathcal{Q}_{2}(X(G))\right), \\
& \Delta(X(G))=\alpha^{2}(X(G))-4 \beta(X(G)) .
\end{aligned}
$$

We introduce a condition

$$
\operatorname{gcd}\{\alpha(X(G))-\sqrt{\Delta(X(G))}, \alpha(X(G))+\sqrt{\Delta(X(G))}\}=1 .
$$

Condition $(\star)$ is an invariant of topological conjugacy.

LEMMA 4.2. Let

$$
G=G_{\mathcal{R}}\left(\{\mathfrak{P}\}, \mathcal{E}^{-} \cup \mathcal{E}^{+}\right), \quad \mathfrak{P}=\{\mathfrak{p}, \mathfrak{q}\},
$$

be an $\mathcal{R}$-graph that satisfies Conditions (I), (II) and (III) such that

$$
\mathfrak{P}_{\mathcal{R}}^{(1)}=\{\mathfrak{q}\},
$$

and that also satisfies Condition $(\star)$.

Then either $\alpha(X(G))-\sqrt{\Delta(X(G))}$ or $\alpha(X(G))+\sqrt{\Delta(X(G))}$ is a divisor of $\rho^{-}(X(G)) \rho^{+}(X(G))$.

Proof. One has that

$$
\begin{aligned}
\alpha(X(G)) & \left.=\operatorname{card}\left(\mathcal{E}^{-}(\mathfrak{p}, \mathfrak{q})\right) \operatorname{card}\left(\mathcal{E}^{+}(\mathfrak{p}, \mathfrak{q})\right)+\sum_{\left(\widehat{e}^{-}, \widehat{e}^{+}\right) \in \widehat{R}} \operatorname{card}\left(\widehat{e}^{-}\right) \operatorname{card}\left(\widehat{e}^{+}\right)\right), \\
\beta(X(G)) & =\operatorname{card}\left(\mathcal{E}^{-}(\mathfrak{p}, \mathfrak{q})\right) \operatorname{card}\left(\mathcal{E}^{+}(\mathfrak{p}, \mathfrak{q})\right)\left(\sum_{\left(\widehat{e}^{-}, \widehat{e}^{+}\right) \in \widehat{R}} \operatorname{card}\left(\widehat{e}^{-}\right) \operatorname{card}\left(\widehat{e}^{+}\right)\right) \\
& \geq \rho^{-}(X(G)) \rho^{+}(X(G)) \operatorname{card}(\widehat{R})>\rho^{-}(X(G)) \rho^{+}(X(G)) .
\end{aligned}
$$

One sees that $\operatorname{card}\left(\mathcal{E}^{-}(\mathfrak{p}, \mathfrak{q})\right) \operatorname{card}\left(\mathcal{E}^{+}(\mathfrak{p}, \mathfrak{q})\right)$ is a root of the equation $z^{2}-\alpha(X(G)) z+$ $\beta(X(G))=0$, which does divide $\rho^{-}(X(G)) \rho^{+}(X(G))$. It also follows from $(\star)$ that

$$
\frac{\rho^{-}(X(G)) \rho^{+}(X(G))}{(\alpha(X(G))-\sqrt{\Delta(X(G))})(\alpha(X(G))+\sqrt{\Delta(X(G))})}=\frac{\rho^{-}(X(G)) \rho^{+}(X(G))}{4 \operatorname{card}\left(\mathcal{Q}_{2}(X(G))\right)}<\frac{1}{4} .
$$

For $\mathcal{R}$-graphs $G$ that satisfy the hypothesis of Lemma 4.2, we set

$$
\begin{aligned}
& \gamma(X(G)) \\
& \quad= \begin{cases}\alpha(X(G))-\sqrt{\Delta(X(G))} & \text { if } \alpha(X(G))-\sqrt{\Delta(X(G))} \mid \rho^{-}(X(G)) \rho^{+}(X(G)), \\
\alpha(X(G))+\sqrt{\Delta(X(G))} & \text { if } \alpha(X(G))+\sqrt{\Delta(X(G))} \mid \rho^{-}(X(G)) \rho^{+}(X(G)) .\end{cases}
\end{aligned}
$$

We say that a relation is indecomposable if it is not isomorphic to a direct sum of relations. We define a family $\mathbf{F}$ of $\mathcal{R}$-graphs $G$ as the family of $\mathcal{R}$-graphs that satisfy the hypothesis of Lemma 4.2 that are such that the relation $\mathcal{Q}_{2}(X(G))$ is indecomposable and that also satisfy the following condition:

$$
\gamma(X(G))=\rho^{-}(X(G)) \rho^{+}(X(G)) .
$$

Condition $(\star \star)$ is an invariant of topological conjugacy. 
LEMMA 4.3. For $\mathcal{R}$-graphs $G=G_{\mathcal{R}}\left(\{\mathfrak{p}, \mathfrak{q}\}, \mathcal{E}^{-} \cup \mathcal{E}^{+}\right)$in the family $\mathbf{F}$, one has that

$$
\operatorname{card}\left(\mathcal{E}^{-}(\mathfrak{p}, \mathfrak{q})\right)=\rho^{-}(X(G)), \quad \operatorname{card}\left(\mathcal{E}^{+}(\mathfrak{p}, \mathfrak{q})\right)=\rho^{+}(X(G))
$$

Proof. One has that

$$
\begin{aligned}
& \rho^{-}(X(G))=\operatorname{card}\left(\mathcal{E}^{-}(\mathfrak{p}, \mathfrak{q})\right) \operatorname{gcd}\left\{\operatorname{card}(\widehat{e}): \widehat{e} \in \widehat{\mathcal{E}}^{-}\right\}, \\
& \rho^{+}(X(G))=\operatorname{card}\left(\mathcal{E}^{+}(\mathfrak{p}, \mathfrak{q})\right) \operatorname{gcd}\left\{\operatorname{card}\left(\widehat{e}^{+}\right): \widehat{e}^{+} \in \widehat{\mathcal{E}}^{+}\right\}
\end{aligned}
$$

and therefore

$$
\begin{aligned}
& \gamma(X(G)) \\
& \quad=\operatorname{card}\left(\mathcal{E}^{-}(\mathfrak{p}, \mathfrak{q})\right) \operatorname{card}\left(\mathcal{E}^{+}(\mathfrak{p}, \mathfrak{q})\right) \operatorname{gcd}\left\{\operatorname{card}(\widehat{e}): \widehat{e}^{-} \in \widehat{\mathcal{E}}^{-}\right\} \operatorname{gcd}\left\{\operatorname{card}(\widehat{e}): \widehat{e}^{+} \in \widehat{\mathcal{E}}^{+}\right\} .
\end{aligned}
$$

By Lemma 4.2, $\gamma(X(G))$ is equal to the root of the equation $z^{2}-\alpha(X(G)) z+$ $\beta(X(G))=0$ that divides $\rho^{-}(X(G)) \rho^{+}(X(G))$. One has that this root is equal to $\operatorname{card}\left(\mathcal{E}^{-}(\mathfrak{p}, \mathfrak{q})\right) \operatorname{card}\left(\mathcal{E}^{+}(\mathfrak{p}, \mathfrak{q})\right)$ and it follows that

$$
\operatorname{gcd}\left\{\operatorname{card}\left(\widehat{e}^{-}\right): \widehat{e}^{-} \in \widehat{\mathcal{E}}^{-}\right\}=\operatorname{gcd}\left\{\operatorname{card}\left(\widehat{e}^{+}\right): \widehat{e}^{+} \in \widehat{\mathcal{E}}^{+}\right\}=1 .
$$

\section{THEOREM 4.4. Let}

$$
G=G_{\mathcal{R}}\left(\{\mathfrak{p}, \mathfrak{q}\}, \mathcal{E}^{-} \cup \mathcal{E}^{+}\right)
$$

be an $\mathcal{R}$-graph that belongs to the family $\mathbf{F}$ and let $\widetilde{G}$ be an $\mathcal{R}$-graph such that the subshifts $X(G)$ and $X(\widetilde{G})$ are topologically conjugate. Then the $\mathcal{R}$-graphs $\widetilde{G}$ and $G$ are isomorphic.

Proof. From the hypothesis that the subshifts $X(G)$ and $X(\widetilde{G})$ are topologically conjugate, it follows by Theorem 2.2 that $\widetilde{G}$ satisfies Conditions (I), (I) and (III). It also follows that

$$
\left\{\Lambda\left(\widehat{e}^{-}\right): \widehat{e}^{-} \in \widehat{\mathcal{E}}^{-}\right\} \subset\{1,2\}
$$

and that

$$
\left\{\widehat{e}^{-} \in \widehat{\mathcal{E}}^{-}: \Lambda\left(\widehat{e}^{-}\right)=2\right\} \neq \emptyset .
$$

At this stage one sees that there is a $K \in \mathbb{Z}_{+}$such that $\widetilde{G}$ appears as an $\mathcal{R}$-graph

$$
\widetilde{G}=G_{\widetilde{\mathcal{R}}}\left(\widetilde{\mathfrak{P}}, \widetilde{\mathcal{E}}^{-} \cup \widetilde{\mathcal{E}}^{+}\right), \quad \widetilde{\mathfrak{P}}=\{\widetilde{p}, \widetilde{q}\} \cup\left\{\widetilde{q}_{k}: 1 \leq k \leq K\right\},
$$

such that

$$
\widetilde{\mathfrak{P}}_{\widetilde{R}}^{(1)}=\{\widetilde{q}\} \cup\left\{\widetilde{q}_{k}: 1 \leq k \leq K\right\} .
$$

One notes that the relation $\mathcal{Q}_{2}(X(\widetilde{G}))$ is indecomposable precisely if $K=0$ and the relation $\widetilde{R}(\widetilde{\mathfrak{q}}, \widetilde{\mathfrak{p}})$ is indecomposable. It follows that $\widetilde{G}$ belongs to the family $\mathbf{F}$.

From the hypothesis that the subshifts $X(G)$ and $X(\widetilde{G})$ are topologically conjugate, it follows by [Kr4, Theorem 2.1] that the $\mathcal{R}$-graphs $\widehat{\widetilde{G}}$ and $\widehat{G}$ are isomorphic. It also follows that

$$
\rho^{-}(X(\widetilde{G}))=\rho^{-}(X(G)), \quad \rho^{+}(X(\widetilde{G}))=\rho^{+}(X(G))
$$

and, by Lemma 4.3, one has that

$$
\operatorname{card}\left(\mathcal{E}^{-}(\tilde{\mathfrak{p}}, \tilde{\mathfrak{q}})\right)=\operatorname{card}\left(\mathcal{E}^{-}(\mathfrak{p}, \mathfrak{q})\right), \quad \operatorname{card}\left(\mathcal{E}^{+}(\widetilde{\mathfrak{p}}, \tilde{\mathfrak{q}})\right)=\operatorname{card}\left(\mathcal{E}^{+}(\mathfrak{p}, \mathfrak{q})\right)
$$


The proof is completed by observing that, for the $\mathcal{R}$-graphs $G$ in the family $\mathbf{F}$, the relation $\mathcal{R}(\mathfrak{p}, \mathfrak{p})$ is isomorphic to the relation $\mathcal{Q}_{1}(X(G))$, and the relation $\mathcal{R}(\mathfrak{q}, \mathfrak{p})$ can be obtained from the the triple that consists of the relation $\widehat{\mathcal{R}}$ and the pair of mappings

$$
\begin{gathered}
\widehat{e} \rightarrow \operatorname{card}(\widehat{e})=\frac{\operatorname{card}\left(P_{2}^{\left(\widehat{e}^{-}\right)}\right)}{\operatorname{card}\left(\mathcal{E}^{-}(\mathfrak{p}, \mathfrak{q})\right)}\left(\widehat{e} \in \widehat{\mathcal{E}}^{-}\right), \\
\widehat{e} \rightarrow \operatorname{card}\left(\widehat{e}^{-}\right)=\frac{\operatorname{card}\left(P_{2}^{\left(\widehat{e}^{+}\right)}\right)}{\operatorname{card}\left(\mathcal{E}^{+}(\mathfrak{p}, \mathfrak{q})\right)}\left(\widehat{e}^{+} \in \widehat{\mathcal{E}}^{+}\right)
\end{gathered}
$$

and by noting that this triple is also an invariant of the topological conjugacy of $X(G)$.

Acknowledgement. Thanks go to the referee for valuable critical remarks.

\section{REFERENCES}

[AH] C. J. Ash and T. E. Hall. Inverse semigroups on graphs. Semigroup Forum 11 (1975), 140-145.

[BBD1] M.-P. Béal, M. Blockelet and C. Dima. Finite-type-Dyck shift spaces. Preprint, 2013. arXiv:1311.4223.

[BBD2] M.-P. Béal, M. Blockelet and C. Dima. Sofic-Dyck shifts. Proc. Int. Symp. on Mathematical Foundations of Computer Science 2014, Part I (Lecture Notes in Computer Science, 8634). Springer, Berlin, 2014, pp. 63-74.

[BBD3] M.-P. Béal, M. Blockelet and C. Dima. Sofic-Dyck shifts. Theoret. Comput. Sci. 609 (2016), $226-244$.

[CK] J. Cuntz and W. Krieger. A class of $C^{*}$-algebras and topological Markov chains. Invent. Math. 56 (1980), 251-268.

[CS] A. Costa and B. Steinberg. A categorical invariant of flow equivalence of shifts. Ergod. Th. \& Dynam. Sys. 36 (2016), 470-513.

[HI] T. Hamachi and K. Inoue. Embeddings of shifts of finite type into the Dyck shift. Monatsh. Math. 145 (2005), 107-129.

[HIK] T. Hamachi, K. Inoue and W. Krieger. Subsystems of finite type and semigroup invariants of subshifts. J. Reine Angew. Math. 632 (2009), 37-61.

[HK1] T. Hamachi and W. Krieger. On certain subshifts and their associated monoids. Ergod. Th. \& Dynam. Sys. 36 (2016), 96-107.

[HK2] T. Hamachi and W. Krieger. Families of directed graphs and topological conjugacy of the associated Markov-Dyck shifts. Preprint, 2018. arXiv:1806.09051.

[Ki] B. P. Kitchens. Symbolic Dynamics. Springer, Berlin, 1998.

[KM1] W. Krieger and K. Matsumoto. A notion of synchronization of symbolic dynamics and a class of C*-algebras. Acta Appl. Math. 126 (2013), 263-275.

[KM2] W. Krieger and K. Matsumoto. Markov-Dyck shifts, neutral periodic points and topological conjugacy. Discrete Contin. Dyn. Syst. 39 (2019), 1-18.

[Kr1] W. Krieger. On the uniqueness of the equilibrium state. Math. Systems Theory 8 (1974), 97-104.

[Kr2] W. Krieger. On a syntactically defined invariant of symbolic dynamics. Ergod. Th. \& Dynam. Sys. 20 (2000), 501-516.

[Kr3] W. Krieger. On flow-equivalence of $\mathcal{R}$-graph shifts. Münster J. Math. 8 (2015), 229-239.

[Kr4] W. Krieger. On subshift presentations. Ergod. Th. \& Dynam. Sys. 37 (2017), 1253-1290.

[L] M. V. Lawson. Inverse Semigroups. World Scientific, Singapore, 1998.

[LM] D. Lind and B. Marcus. An Introduction to Symbolic Dynamics and Coding. Cambridge University Press, Cambridge, 1995.

[M] K. Matsumoto. C*-algebras arising from Dyck systems of topological Markov chains. Math. Scand. 109 (2011), 31-54.

[NP] M. Nivat and J.-F. Perrot. Une généralisation du monoide bicyclique. C. R. Acad. Sci. Paris Ser. A 271 (1970), 824-827. 